
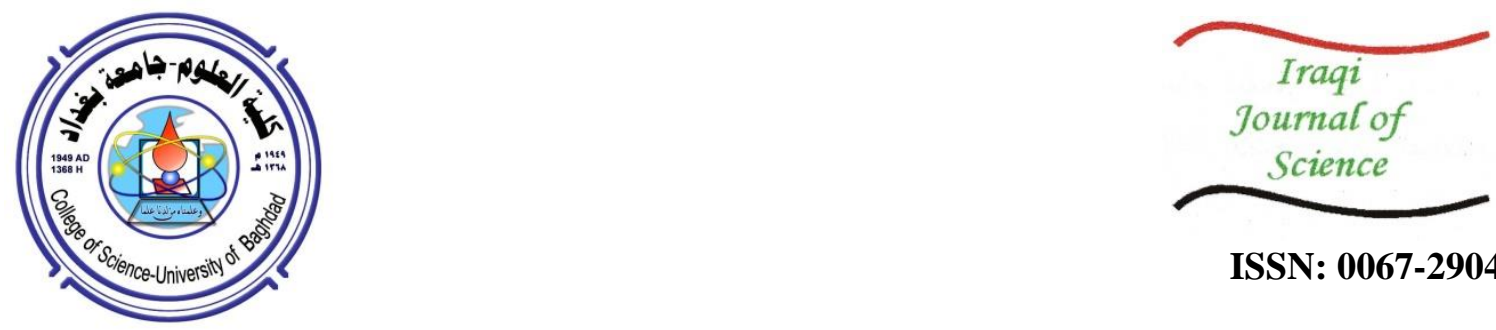

ISSN: 0067-2904

\title{
A Numerical Study on Cross Diffusion Cattaneo-Christov Impacts of MHD Micropolar Fluid Across a Paraboloid
}

\author{
K. Kalyani ${ }^{1}$, N. Seshagiri Rao ${ }^{2}$, M.V.V.N.L. Sudha Rani ${ }^{3}$ \\ ${ }^{\mathbf{1}}$ Department of Mathematics, Vignan's Foundation for Science, Technology \& Research, Vadlamudi-522213, \\ Andhra Pradesh, India. \\ ${ }^{2}$ Department of Applied Mathematics, School of Applied Natural Sciences, Adama Science and Technology \\ University, Post Box No.1888, Adama, Ethiopia. \\ ${ }^{3}$ Department of Mathematics, Acharya Nagarjuna University, Ongole Campus, Ongole, Andhra Pradesh, India
}

Received: $17 / 5 / 2020$

Accepted: $31 / 8 / 2020$

\begin{abstract}
Analyzing the impacts of Cattaneo-Christov flux, bioconvective Raleigh number and cross diffusion effects in electrically conducting micropolar fluid through a paraboloid revolution is assessed in this work. Non-dimensional equations are solved numerically using shooting technique with an aid of Matlab software. The impact of various parameters on velocity, temperature and concentration are discussed in detail and presented graphically. Harman number and micro rotation parameters are found and have an increasing influence on shear stress. The vertical velocity increases at free stream and the horizontal velocity increases near the surface when $\mathrm{Gr}_{\mathrm{b}}$ increases, which follows the opposite trend for accumulation of $R_{b}$. The numerical results are compared with the available data indicating good agreement in a limiting case.
\end{abstract}

Key words: Micropolar fluid, Cattaneo-Christov heat flux, Paraboloid surface, thermal radiation, Diffusion effects.

\section{Introduction}

The flow characteristics based on some variants such as liquid crystals, extrusion of polymer fluids, cooling of a metallic plate in a bath, exoteric lubricants, and colloidal interruption cannot be analyzed by the approach of Navier Stokes equations of Newtonian and Non-Newtonian fluids. Many researchers constructed many constitutive statements. Out of these, the micro polar fluid model was introduced by Eringen [1]. Micro polar fluid is a fluid which consists of a micro-structure that is applicable to a group of fluids along with unsymmetrical stress tensor. The analysis of micro polar fluid has attracted its diverse mechanical and manufacturing applications. Das [2] analyzed the importance of radiation specification on Magneto hydrodynamics (MHD) micropolar fluid through the reference of rotating frame. Dulal Pal and Chatterjee [3] exhibited the 2D micropolar non-Darcian flow with thermal radiation and non-uniform heat source through a permeable stretching panel with boundary layer, and employed analytically with Finite difference method (FDM). Gangadhar et al. [4] analyzed numerically the consequences of the slip effect on MHD micropolar fluid and explained the enhancement of temperature profiles with respect to the accumulation of slip factor. Ghadikolaei et al. [5] investigated the influence of $\mathrm{TiO} 2$ water nanoparticles on micropolar flow including dust particles.

*Email: kalyani.namana@gmail.com 
Gul et al. [6] presented Homotopy Analysis Method HAM to solve the heat transfer of a liquid film of a micropolar flow through a porous medium and also used ND solving technique to get numerical solutions. Impact of heat source /sink on micropolar-free convective fluid flow which passes through a shrinking panel was reported by Mishra et al. [7]. Qasim et al. [8] discussed heat transfer in a micropolar fluid over a stretching sheet with Newtonian heat source. Aurangzaib et al. [9] tested the importance of heat transfer of micropolar fluid flow over an exponentially permeable stretching sheet. In an enclosed MHD the boundary layer flow, heat and mass transmission possess an extensive attention in industrial appliances such as polymer and metal suspension, dispose of chemical material, exchangers, etc. The phenomena of transferring heat and mass play an extensive role in controlling the quality of the output in the outflows. The transformation of temperature and concentration in MHD flows also has essential applications which include magnetic substantial processing, control of the cooling rate, geophysics, etc. Kumaran et al. [10] investigated the temperature displacement on two non-Newtonian fluid flows across a paraboloid revolution and presented graphical representations of both carreau and casson fluids. Khan et al. [11] studied the bioconvection flow of a carreau fluid by a paraboloid revolution with the aid of an implicit finite difference technique (Keller-Box). The boundary layer analysis of Blasius flow of Carreau fluid on an upper horizontal surface of paraboloid of revaluation (UHSPR) was deliberated by Animasaun and Pop [12]. Abegunrin et al. [13] discussed the comparison between two non-Newtonian fluids (Casson and Williamson fluids) over an UHSPR. Animasaun [14] explored the temperature and concentration displacements about nanofluid flows across a paraboloid revolution. Rehman et al. [15] studied the physical phenomena of both the Newtonian and non-Newtonian fluid models, which constitute a set of complex mathematical equations. Vishnu Ganesh et al. [16] analyzed heat transfer and the effects on hydromagnetic nanofluid across a melting surface. Khalil et al. [17] presented the magneto hydrodynamic (MHD) Prandtl-Eyring nanofluid over a stretching surface with new mass flux conditions. Khalil et al. [18] implemented a hydromagnetic non-Newtonian fluid across a stretching cylinder. Ramzan et al. [19] investigated heat and mass transfer and, the impact on hydromagnetic Jeffery nanofluid across a stretching cylinder and also observed the exchange of heat transfer between two objects or within the object is a substantial phenomenon in the nature. Cattaneo [20] introduced the attitude of classical Fourier's law, which was examined by thermal relaxation time. Later Christov [21] implemented the upper convected Oldroyd's derivatives along with thermal relaxation time for attaining the formulation for material-invariant. Straughan [22] addressed the flow of viscous fluid in the presence of CattaneoChristov effect. A study of three dimensional (3D) flows of heat conduction and mass diffusion done by Hayat et al. [23]. They computed the analytical solutions of governing flow system of equations by applying OHAM. A theoretical inspection of an unsteady flow and temperature displacement of dusty nanofluid was presented by Mamatha et al. [24] and also provided the graphical representations of both dusty nanofluid and dusty fluid. Ramzan et al. [25] studied the 3D upper convected Maxwell fluid across a stretching surface and obtained a solution using MATLAB software. Ramzan et al. [26] analyzed the effect of Cattaneo-Christov heat flux with 3D non-Newtonian Maxwell fluid. Ramzan et al. [27] explored the numerical solution of two dimensional MHD stagnation point in Williamson fluid under the influence of homogeneous-heterogeneous reactions over a linearly stretched surface. Soret and Dufour effects on magneto hydrodynamic Oldroyd-B fluid across a cone/wedge were analyzed by Reddy [28] using Matlab. In the recent past, the influences of Cattaneo-Christov, thermal radiation, heat source on boundary layer flow and heat transfer were widely examined in many articles [29-40]. Many investigators concentrate on analyzing the transformation of heat and mass transfer in MHD flows. However, in the present model, the effects of MHD micropolar fluid across a paraboloid in the direction of Cattaneo-Christov heat flux along with the impacts of cross diffusion effects are evaluated. The mathematical model of the problem and the method to obtain the solution are explained in section 2. The results and discussion of the problem are analyzed in Section 3. Concluding remarks are given in section 4.

\section{Mathematical formulation}

Consider the two-dimensional (2D) micropolar mixed convection flow over a paraboloid revolution. $A(X+b)^{\frac{m-1}{2}} \leq Y \leq \infty$ is the region of flow (here $\mathrm{A}$ and $\mathrm{b}$ are positive and $m<1$ ) and 
$U_{w}=U_{0}(X+b)^{m}$ is the stretched parallel with the velocity of the imminent fluid layers on the surface. Here the horizontal surface is placed along $\mathrm{X}$-axis, while $\mathrm{Y}$-axis is perpendicular to it.

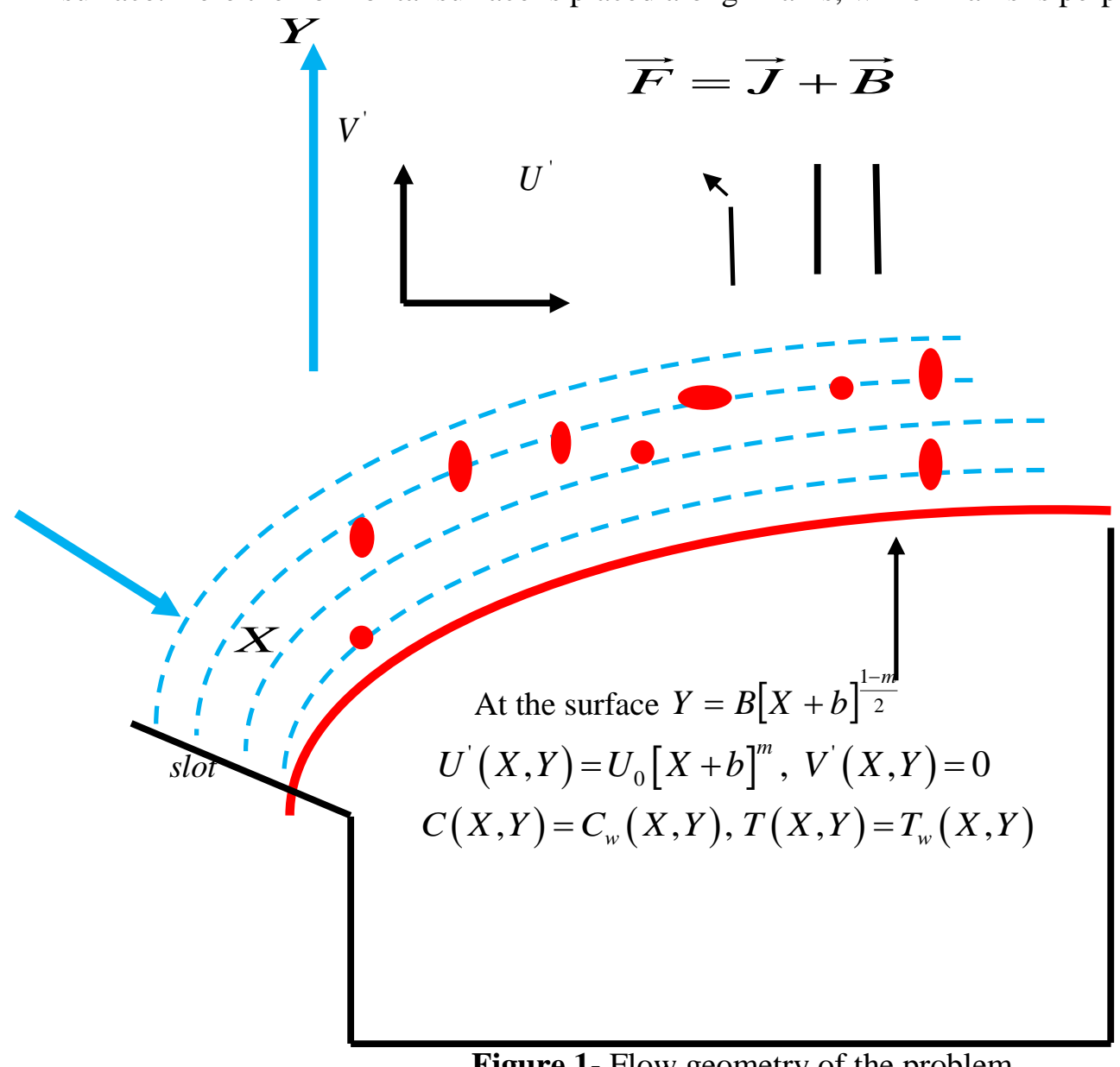

Figure 1- Flow geometry of the problem

The suitable governing equations to explore the micropolar fluid of a boundary layer flow passing through paraboloid revolution are given by

$$
\begin{aligned}
& \frac{\partial U^{\prime}}{\partial X}+\frac{\partial V^{\prime}}{\partial Y}=0 \\
& U^{\prime} \frac{\partial U^{\prime}}{\partial X}+V^{\prime} \frac{\partial U^{\prime}}{\partial Y}=\left(v+\frac{k}{\rho}\right) \frac{\partial^{2} U^{\prime}}{\partial X^{2}}+\frac{k}{\rho} \frac{\partial N^{\prime}}{\partial Y}+\frac{\partial}{\partial X}\left(g \beta_{X}\left(\frac{m+1}{2}\right)\left(T-T_{\infty}\right)\right) \\
& +\frac{\partial}{\partial X}\left(g \beta^{*}{ }_{X}\left(\frac{m+1}{2}\right)\left(C-C_{\infty}\right)\right)-\frac{\sigma B_{0}^{2}}{\rho} U^{\prime} \\
& U^{\prime} \frac{\partial U^{\prime}}{\partial X}+V^{\prime} \frac{\partial V^{\prime}}{\partial Y}=\frac{\gamma^{*}}{\rho j} \frac{\partial^{2} N^{\prime}}{\partial Y^{2}}-\frac{k}{\rho j}\left(2 N^{\prime}+\frac{\partial U^{\prime}}{\partial Y}\right), \\
& U^{\prime} \frac{\partial T}{\partial X}+V^{\prime} \frac{\partial T}{\partial Y}+\lambda_{1}\left(\begin{array}{l}
U^{\prime} \frac{\partial U^{\prime}}{\partial X} \frac{\partial T}{\partial X}+V^{\prime} \frac{\partial V^{\prime}}{\partial Y} \frac{\partial T}{\partial Y}+U^{\prime} \frac{\partial V^{\prime}}{\partial X} \frac{\partial T}{\partial Y}+V^{\prime} \frac{\partial U^{\prime}}{\partial Y} \frac{\partial T}{\partial X} \\
+2 U^{\prime} V^{\prime} \frac{\partial^{2} T}{\partial X \partial Y}+U^{\prime 2} \frac{\partial^{2} T}{\partial X^{2}}+V^{\prime 2} \frac{\partial^{2} T}{\partial Y^{2}}
\end{array}\right) \\
& =\frac{k_{1}}{\rho c_{p}} \frac{\partial^{2} T}{\partial Y^{2}}-\frac{1}{\rho c_{p}} \frac{\partial q_{r}}{\partial Y}+\frac{\rho D_{m} K_{t}}{c_{s} c_{p}} \frac{\partial^{2} C}{\partial Y^{2}} \\
& U^{\prime} \frac{\partial C}{\partial X}+V^{\prime} \frac{\partial C}{\partial Y}=D_{m} \frac{\partial^{2} C}{\partial Y^{2}}+\frac{D_{m} K_{t}}{T_{m}} \frac{\partial^{2} T}{\partial Y^{2}} \text {. }
\end{aligned}
$$


The boundary conditions of the problem are

$$
\begin{gathered}
U^{\prime}\left(X, A(X+b)^{\frac{1-m}{2}}\right)=U_{w}(X)=U_{0}(X+b)^{m}, \quad V^{\prime}\left(X, A(X+b)^{\frac{1-m}{2}}\right)=0, \\
N^{\prime}\left(X, A(X+b)^{\frac{1-m}{2}}\right)=-m_{0} \frac{\partial U^{\prime}}{\partial Y}, \quad T=T_{w}, \quad C=C_{w} \text { at } Y=A(X+b)^{\frac{1-m}{2}}, \\
U^{\prime} \rightarrow 0, N^{\prime} \rightarrow 0, T \rightarrow T_{\infty}, C \rightarrow C_{\infty} \text { as } Y \rightarrow \infty,
\end{gathered}
$$

where $U^{\prime}$ and $V^{\prime}$ are expressed as the velocities along the directions $X$ and $Y ; k, \mu$ and $v$ are known as the vortex, dynamic and kinematic viscosities respectively; $\rho$ and $g$ are the density of the fluid and the gravitational acceleration; $\beta$ and $\beta^{*}$ are the thermal and solutal expansion coefficients; $\gamma^{*}=\left(\mu+\frac{k}{2}\right) j$ is the spin gradient viscosity; $\alpha=\frac{k_{1}}{\rho c_{p}}$ is the thermal diffusivity; $N$ is the micro rotation velocity; $j=v / a$ is the micro inertia; $c_{p}, m_{0}, \lambda_{1}$ and $k_{1}$ are the specific heat at constant pressure, boundary parameter, heat flux at relaxation time and the fluid thermal diffusivity respectively; $T_{\infty}$ and $C_{\infty}$ are temperature and concentration of the ambient fluid.

The similarity transformations are as follows

$$
\begin{aligned}
& U^{\prime}=U_{0}(X+b)^{m} F^{\prime}(\xi), \quad V^{\prime}=-\sqrt{\frac{(m+1) u_{0}(X+b)^{m-1}}{2}}\left[F(\xi)+\xi \frac{m-1}{m+1} F^{\prime}(\xi)\right], \\
& \psi=\sqrt{\frac{2 v U_{0}(Y+b)^{m+1}}{m+1} F(\xi), \quad \xi=\sqrt{\frac{(m+1) U_{0}(X+b)^{m-1}}{2 v}} y,} \\
& N^{\prime}=U_{0}(X+b)^{m} \sqrt{\frac{U_{0}(m+1)}{2 v}}(X+b)^{\frac{m-1}{2}} H(\xi), \quad \theta(\xi)=\frac{T-T_{\infty}}{T_{w}-T_{\infty}}, \varphi(\xi)=\frac{C-C_{\infty}}{C_{w}-C_{\infty}} .
\end{aligned}
$$

Using the transform (7), the equations (2)-(6) are transformed as follows:

$$
\begin{aligned}
& (1+K) F^{\prime \prime \prime}+K H^{\prime}+F F^{\prime \prime}-(2 m /(m+1)) F^{\prime 2}+G r_{b} \theta+R_{b} \varphi-H a F^{\prime}=0, \\
& (1+(K / 2)) H^{\prime \prime}+F H^{\prime}-((3 m-1) /(m+1)) F^{\prime} H-(2 K /(m+1))\left(2 H+F^{\prime \prime}\right)=0, \\
& F \theta^{\prime}+(1+(4 / 3) N r)\left(\theta^{\prime \prime} / \operatorname{Pr}\right)+D u \varphi^{\prime \prime}-\gamma((m-1) / 2)\left[\begin{array}{l}
(3-m) /(m+1) F F^{\prime} \theta^{\prime}+ \\
((m-1) / 2) F^{2} \theta^{\prime \prime}
\end{array}\right]=0, \\
& (1+K) F^{\prime \prime \prime}+K H^{\prime}+F F^{\prime \prime}-(2 m /(m+1)) F^{\prime 2}+G r_{b} \theta+R_{b} \varphi-H a F^{\prime}=0,
\end{aligned}
$$

together with the boundary conditions:

$$
\begin{gathered}
F(\alpha)=\alpha(1-m) /(1+m), F^{\prime}(\alpha)=1, H(\alpha)=-m_{0} F^{\prime \prime}(\alpha), \theta(\alpha)=1, \varphi(\alpha)=1 \text { at } \alpha=0, \\
F^{\prime}(\alpha) \rightarrow 0, H(\alpha) \rightarrow 0, \theta(\alpha) \rightarrow 0, \varphi(\alpha) \rightarrow 0 \text { as } \alpha \rightarrow \infty,
\end{gathered}
$$

here $\alpha=A \sqrt{\frac{U_{0}(m+1)}{2 v}}$ denotes UHSPR.

$F(\zeta-\alpha)=f(\eta), H(\zeta-\alpha)=h(\eta), \Theta(\zeta-\alpha)=\theta(\eta), \Phi(\zeta-\alpha)=\phi(\eta)$ are used to alter the system as follows:

$$
\begin{aligned}
& (1+K) f^{\prime \prime \prime}+K f^{\prime}+f f^{\prime \prime}-(2 m /(m+1))+f^{\prime 2}+G r_{b} \theta+R_{b} \phi-H a f^{\prime}=0, \\
& (1+K / 2) h^{\prime \prime}+f h^{\prime}-[(3 m-1) /(m+1)] f^{\prime} h-(2 K /(m+1))\left(2 h+f^{\prime \prime}\right)=0, \\
& f \theta^{\prime}+(1+(4 / 3) N r)\left(\theta^{\prime \prime} / \operatorname{Pr}\right)+D u \varphi^{\prime \prime}=\gamma((m-1) / 2)\left[(3-m) /(m+1) f f^{\prime} \theta^{\prime}+((m-1) / 2) f^{2} \theta^{\prime \prime}\right],
\end{aligned}
$$




$$
\begin{aligned}
& \phi^{\prime \prime}+S c f \phi^{\prime}+S c S r \theta^{\prime \prime}=0, \\
& f(0)=\alpha(1-m) /(1+m), f^{\prime}(0)=1, h(0)=-m_{0} f^{\prime \prime}(0), \theta(0)=1, \varphi(0)=1, \\
& f^{\prime}(\infty) \rightarrow 0, h(\infty) \rightarrow 0, \theta(\infty) \rightarrow 0, \varphi(\infty) \rightarrow 0,
\end{aligned}
$$

where $H a, G r_{b}, R_{b}$ and $K$ are signified as the Hartmann number, Grashof number, bioconvection Rayleigh number and micro rotation parameter respectively. The boundary parameter, thermal radiation parameter and Prandtl number are denoted by $m_{0}, N r$ and $\operatorname{Pr} . \quad \gamma, S c, S r$ and $D u$ are known as the thermal relaxation parameter, Schmidt number, Soret number and Dufour number respectively.

The physical quantities of interest are

$C_{f} \operatorname{Re}_{x}^{1 / 2}=\sqrt{\frac{m+1}{2}} f^{\prime \prime}(0), \quad N u_{x} \operatorname{Re}_{x}^{-1 / 2}=-\sqrt{\frac{m+1}{2}} \theta^{\prime}(0), \quad S h_{x} \operatorname{Re}_{x}^{-1 / 2}=-\sqrt{\frac{m+1}{2}} \phi^{\prime}(0)$,

where $\operatorname{Re}_{x}=\frac{u_{w}(x+b)}{v}$ is the local Reynolds number.

\section{Results and Discussion}

In the present model, a powerful numerical mechanism of R-K fourth order inclusive of shooting approach and BVP5C method are applied to resolve the dimensionless equations of micropolar fluid flow through the parabolid surface. Influence of Hartmann number, thermal and solutal Grashof numbers, radiation parameter, Prandtl number and, Soret and Dufour effects are discussed in this section. The flow parameter values $\mathrm{Ha}=1.0, \mathrm{Gr}_{\mathrm{b}}=0.2, \mathrm{R}_{\mathrm{b}}=0.2, \mathrm{Nr}=0.5, \mathrm{Pr}=0.72, \mathrm{Sc}=0.6, \mathrm{Sr}=$ $0.5, \mathrm{Du}=0.5, \mathrm{~K}=0.1$ and $\gamma=0.1$ are the input to acquire the results for complete production which are otherwise presented as Figure-ures and tables. The results are demonstrated in Figures(2 - 11), Table-1 and Table-2.

The obtained results are agreeing well with available results in the literature [10, 35].

Table -1 Comparison of obtain results of $\boldsymbol{f}^{\prime \prime}(\mathbf{0})$ with [10,35] for $\boldsymbol{D u}=\boldsymbol{S r}=\boldsymbol{K}=\boldsymbol{m}_{\mathbf{0}}=\boldsymbol{\gamma}=\mathbf{0}$.

\begin{tabular}{|c|c|c|c|}
\hline$m$ & Animasun and Sandeep [35] & Kumaran et al. [10] & Present results \\
\hline 0.1 & -0.8671009 & -0.86710092 & -0.86710093 \\
\hline 0.2 & -0.8624053 & -0.8624053 & -0.8624051 \\
\hline 0.3 & -0.8584863 & -0.8584863 & -0.8584863 \\
\hline 0.4 & -0.8481543 & -0.8481543 & -0.8481544 \\
\hline
\end{tabular}

Figure- 2(a) and 2(b) show the deviations of Hartman number on perpendicular and parallel velocity profiles. In general, the increment in Hartmann number generates the Lorentz force which is known as the resistive force. Figure-2(c) exhibits the shear stress on various values of Ha. For different values of $\mathrm{Ha}$, the shear stress increases at an adjacent to the plate but the response is revoked far off from $1 \leq \eta \leq 5$ and therefore no changes in shear stress occur at the free stream. Figure-2(d) represents $h(\eta)$ for different values of Ha. Near the surface of the plate when Ha increases, $h(\eta)$ rises but the result reverses far off from the plate. Correspondingly $h^{\prime}(\eta)$ decreases as Ha increases which is shown in Figure-2(e).

The influence of thermal Grashof number $\left(G r_{b}\right)$ is presented in Figure- 3(a)-3(e) Figure-3 (a) and 3(b) exhibit the perpendicular and parallel velocity profiles for various values of $G r_{b}$. We observed a significant increase in the perpendicular velocity at the free stream. Also, a parallel velocity developed near the surface when $G r_{b}$ rises. Figure-3(c) explains the effect of shear stress for distinct values of 
$G r_{b}$. The shear stress jumps for change of $G r_{b}$ at an adjacent to the plate, but the outcome is an opposite from $1.2 \leq \eta \leq 3.8$. Hence, no changes in shear stress occur away from wall at the free stream. Figures-3(d) and 3(e) represent the effects of $G r_{b}$ on $h(\eta)$ and $h^{\prime}(\eta)$. It is noticed that $h(\eta)$ diminishes as $G r_{b}$ increases.

Figures- 4(a) and 4(b) demonstrate the perpendicular and parallel velocity profiles for divergent values of $R_{b}$. We observed a significant decline in the perpendicular velocity at the free stream and that the parallel velocity diminishes near the surface when $R_{b}$ advances. Figure- 4(c) presents the effect of shear stress for distinct values of $R_{b}$. It elucidates that at adjacency to the plate shear stress decreases for higher values of $R_{b}$, but the result behaves in opposite from $1.8 \leq \eta \leq 6$. Thereafter, no changes in shear stress occur at the free stream away from wall. Figures 4(d) and 4(e) present the effects of $R_{b}$ on $h(\eta)$ and $h^{\prime}(\eta)$. We noticed that $h(\eta)$ reduces and $h^{\prime}(\eta)$ increases near the upper horizontal sphere of the paraboloid revolution for various values of $R_{b}$.

Figures. 5(a) and 5(b) present the variations of micro rotation parameter $(\mathrm{K})$ on perpendicular and parallel velocity profiles. We observed an appreciable development in the perpendicular velocity at the free stream and that the parallel velocity enlarges near the surface when $\mathrm{K}$ builds up. Figure-5(c) introduces the effect of shear stress for distinct values of K. It illustrated that shear stress increase with increase in $\mathrm{K}$ at an adjacency to the plate. However, the result behaves in opposite thereafter. Figureures. 5(d) and 5(e) show the effects of $\mathrm{K}$ on $h(\eta)$ and $h^{\prime}(\eta)$. We noticed that $h(\eta)$ scales down near the surface and that $h^{\prime}(\eta)$ is increased near USHPR, although the response is reversed beyond the surface for accumulating values of $\mathrm{K}$.

The influence of different values of $N r$ on thermal profile is shown in Figures 6(a)-6(b). They show that the thermal boundary layer thickness and temperature are improved for increasing values of $\mathrm{Nr}$. Higher values of $\mathrm{Nr}$ afford more temperature to the fluid flow which generates an increment in temperature and thermal boundary layer thickness. We identify that the thermal gradient enlarges at USHPR for values from $0 \leq \eta<2.5$ and the effect is in opposite far from the plate for higher values of $\mathrm{Nr}$ (Figure-6b).

The impact of $\operatorname{Pr}$ on thermal profile is shown in Figure-7(a). It is illustrated that the thermal profile decreases upon the increase of Pr. Figure-7(b) describes the thermal gradient for various values of $\mathrm{Pr}$ . It is observed that the thermal gradient depreciates near UHSPR, but the impact is opposite far off from the plate when $1.5<\eta<5$, while the thermal gradient difference is not considered thereafter up to the free stream.

The influence of thermal distribution for distinct values of thermal relaxation specification $(\gamma)$ is illustrated in Figure-8(a). It is noticed that the thermal profile is depreciating upon rising $\gamma$. In addition, the thickness of the thermal boundary layer was generally declined when $\gamma$ was increased. Particles of the object need a long extent to shift temperature to their neighboring particles. Especially, we can clarify that for large values of $\gamma$ material that represent a non-conducting attitude which is important in the minimization of thermal profile. Analysis of $\gamma$ on thermal gradient profile is presented in Figure-8(b). The thermal gradient decreases near USHPR and the result is reversal away from the plate.

The influence of the concentration profile for various traits of Schmidt number ( $\mathrm{Sc}$ ) is shown in Figures-9(a) and 9(b). It is indicted from Figure-9(a) that the concentration profile drops rapidly when Sc increased from 1.0 to 1.5 , hence the thickness of the solutal boundary layer decreased. This explains that large diffusing species have more decelerating effects on the concentration profile. Figure-9(b) shows the impact of concentration gradient for distinct values of Sc.

Figure-10(a) and 10(c) reveal the significance of Dufour effect on thermal and concentration profiles. We observe from the Figures the development of thermal profile despite the decline in the concentration profile with an increasing of Dufour effect. The impact of the concentration on the temperature is determined from Dufour effect. The impacts of thermal gradient and concentration 
gradient for various Dufour values are shown in Figures-10(b) and 10(d). The accumulation of thermal gradient and concentration gradient near USHPR are observed, while the effect is reversed away from the plate.

The impact of Soret effect on the concentration profile is shown in Figure-11(a). It is observed that the profile of concentration is increased with the increase of Soret effect as well as the thickness of the boundary layer Figure-11(b) depicts the concentration gradient for a distinct value of Sr. It is revealed that the concentration gradient increases near USHPR, but the effect is reversed from $1.8 \leq \eta \leq 6$ and not considerable change observed till free stream.

Table 2-Variations of $C f_{x}, N u_{x}, S h_{x}$ for different values of all non-dimensional parameters

\begin{tabular}{|c|c|c|c|c|c|c|c|c|c|c|c|c|c|c|}
\hline$H a$ & $G r_{b}$ & $R_{b}$ & $K$ & $\alpha$ & $m_{0}$ & $\operatorname{Pr}$ & $\mathrm{Nr}$ & $\gamma$ & $S r$ & Sc & $D u$ & $C f_{x}$ & $N u_{x}$ & $S h_{x}$ \\
\hline 0.5 & & & & & & & & & & & & -0.092953 & -0.162327 & -0.690284 \\
\hline 1.0 & & & & & & & & & & & & -0.194258 & -0.145504 & -0.660625 \\
\hline 1.5 & & & & & & & & & & & & -0.447605 & -0.130689 & -0.633614 \\
\hline 2.0 & & & & & & & & & & & & -0.673405 & -0.117868 & -0.609084 \\
\hline & 0.1 & & & & & & & & & & & -0.511660 & -0.098064 & -0.604424 \\
\hline & 0.3 & & & & & & & & & & & -0.364789 & -0.120134 & -0.631027 \\
\hline & 0.5 & & & & & & & & & & & -0.227067 & -0.135672 & -0.651480 \\
\hline & & 0.1 & & & & & & & & & & -0.214344 & -0.156899 & -0.668514 \\
\hline & & 0.3 & & & & & & & & & & -0.124867 & -0.158569 & -0.675083 \\
\hline & & 0.5 & & & & & & & & & & -0.036787 & -0.160139 & -0.681359 \\
\hline & & & 0.0 & & & & & & & & & -0.092953 & -0.162327 & -0.690284 \\
\hline & & & 0.5 & & & & & & & & & -0.051145 & -0.170314 & -0.691011 \\
\hline & & & 1.0 & & & & & & & & & -0.006412 & -0.176702 & -0.689981 \\
\hline & & & & 0.1 & & & & & & & & 0.128346 & -0.174519 & -0.650567 \\
\hline & & & & 0.2 & & & & & & & & 0.119834 & -0.171544 & -0.660396 \\
\hline & & & & 0.3 & & & & & & & & 0.111099 & -0.168520 & -0.670292 \\
\hline & & & & & 0.5 & & & & & & & 0.092953 & -0.162327 & -0.690284 \\
\hline & & & & & 1.0 & & & & & & & 0.092953 & -0.162327 & -0.690284 \\
\hline & & & & & 1.5 & & & & & & & 0.092953 & -0.162327 & -0.690284 \\
\hline & & & & & & 0.5 & & & & & & -0.180668 & -0.075360 & -0.618486 \\
\hline & & & & & & 1.0 & & & & & & -0.207972 & -0.413043 & -1.056249 \\
\hline & & & & & & 1.5 & & & & & & -0.702567 & -0.534724 & -1.852004 \\
\hline & & & & & & & 0.5 & & & & & 0.103831 & -0.160158 & -0.693184 \\
\hline & & & & & & & 1.0 & & & & & 0.144408 & -0.151289 & -0.703017 \\
\hline & & & & & & & 1.5 & & & & & 0.170370 & -0.145133 & -0.708649 \\
\hline & & & & & & & & 0.01 & & & & 0.092123 & -0.164470 & -0.688691 \\
\hline & & & & & & & & 0.1 & & & & 0.092953 & -0.162327 & -0.690284 \\
\hline & & & & & & & & 0.5 & & & & 0.096663 & -0.152807 & -0.690645 \\
\hline & & & & & & & & & 0.6 & & & -0.092953 & -0.170559 & -0.690284 \\
\hline & & & & & & & & & 1.2 & & & -0.093636 & -0.162327 & -0.675116 \\
\hline & & & & & & & & & 1.8 & & & -0.012008 & -0.113318 & -0.562597 \\
\hline & & & & & & & & & & 0.6 & & 0.092953 & -0.318078 & -0.690284 \\
\hline & & & & & & & & & & 1.2 & & 0.207881 & -0.162327 & -0.546288 \\
\hline & & & & & & & & & & 1.8 & & 0.803107 & -0.059119 & -0.173318 \\
\hline & & & & & & & & & & & 0.6 & 0.168375 & -0.162327 & -0.468305 \\
\hline & & & & & & & & & & & 1.2 & 0.092953 & -0.507380 & -0.690284 \\
\hline & & & & & & & & & & & 1.8 & 0.064737 & -3.438555 & -0.764152 \\
\hline
\end{tabular}




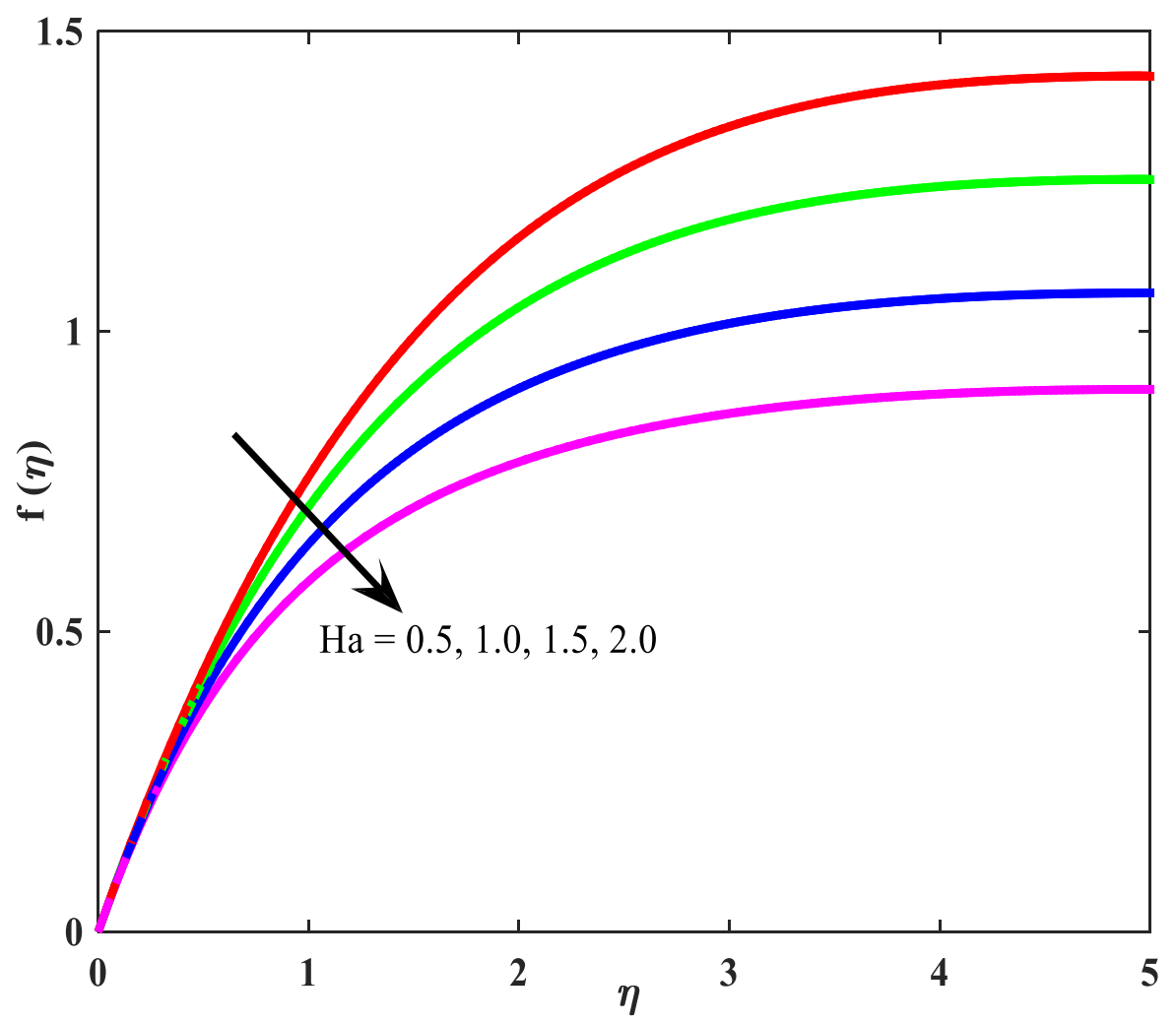

Figure 2(a)-Variations of $H a$ on $f(\eta)$

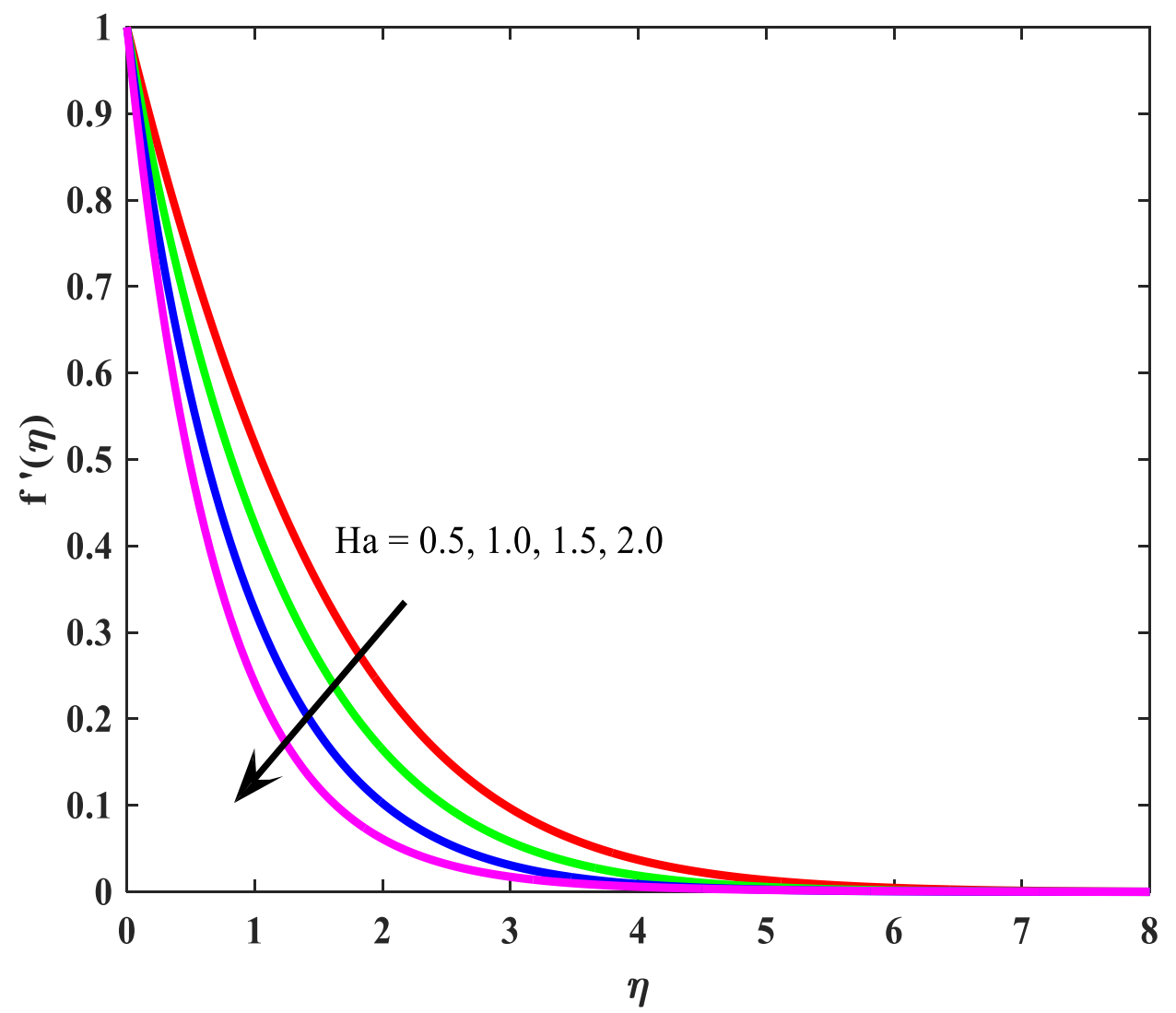

Figure 2(b)-Variations of $H a$ on $f^{\prime}(\eta)$ 


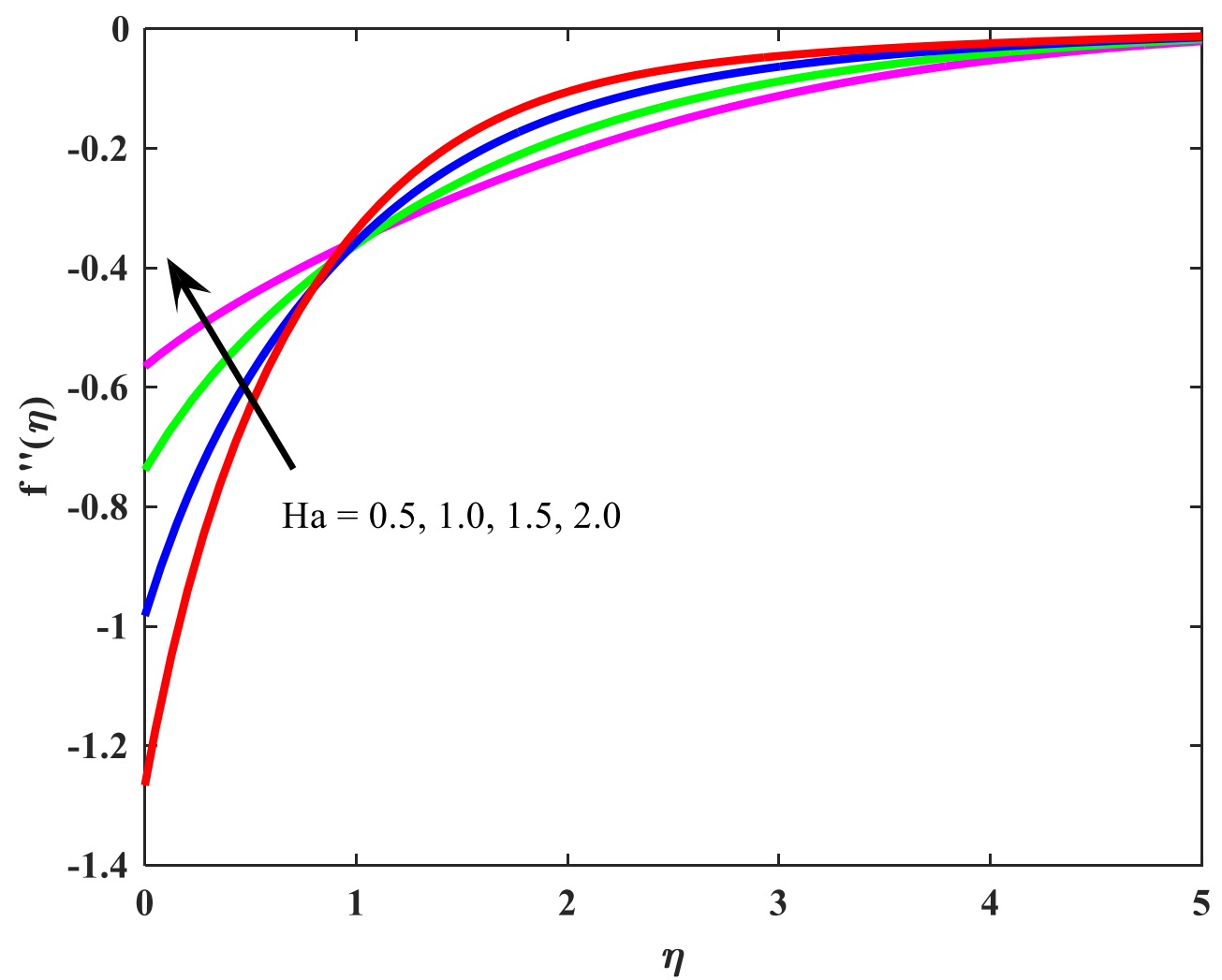

Figure 2(c)-Variations of $H a$ on $f^{\prime \prime}(\eta)$

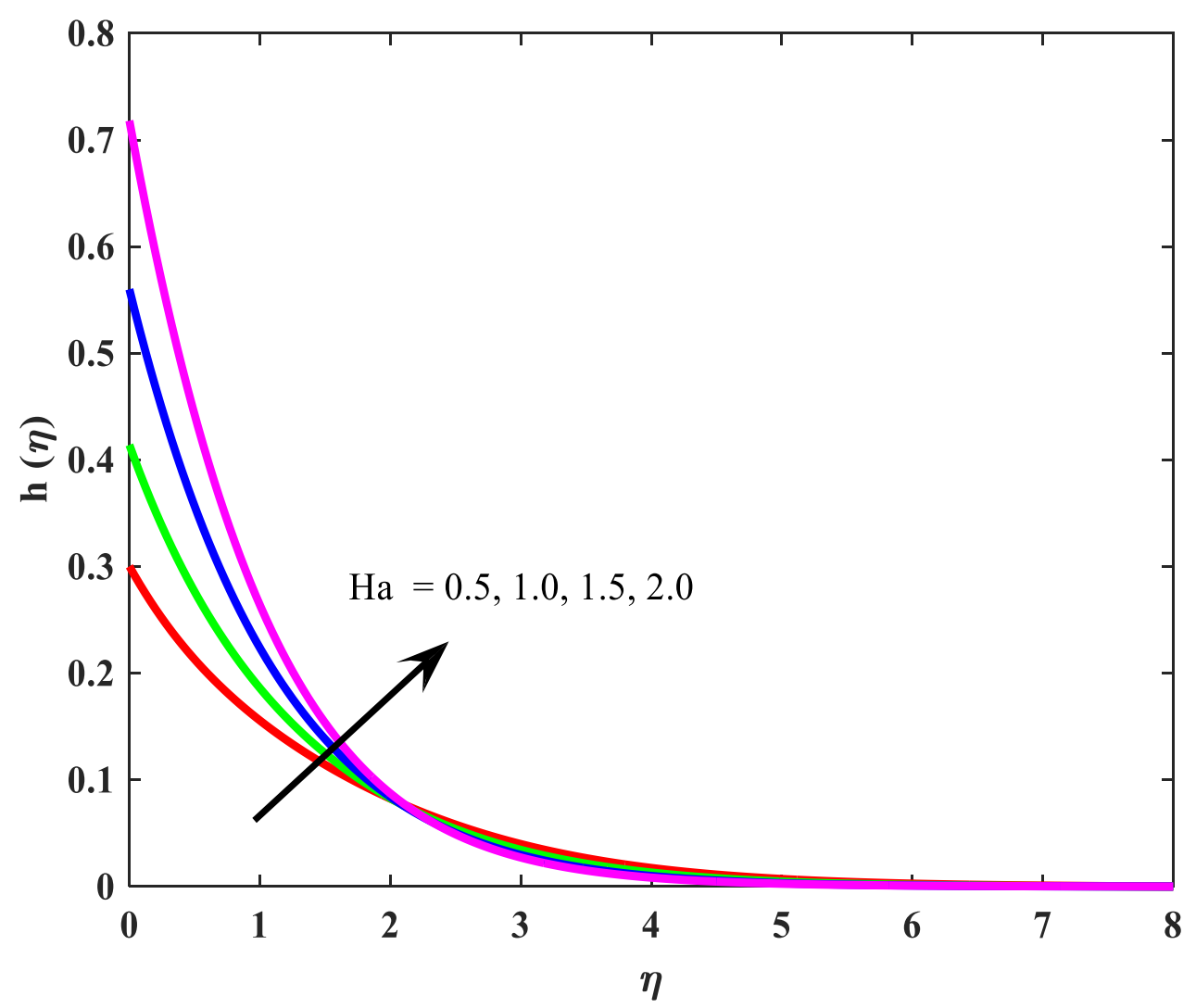

Figure 2(d)-Variations of $H a$ on $h(\eta)$ 


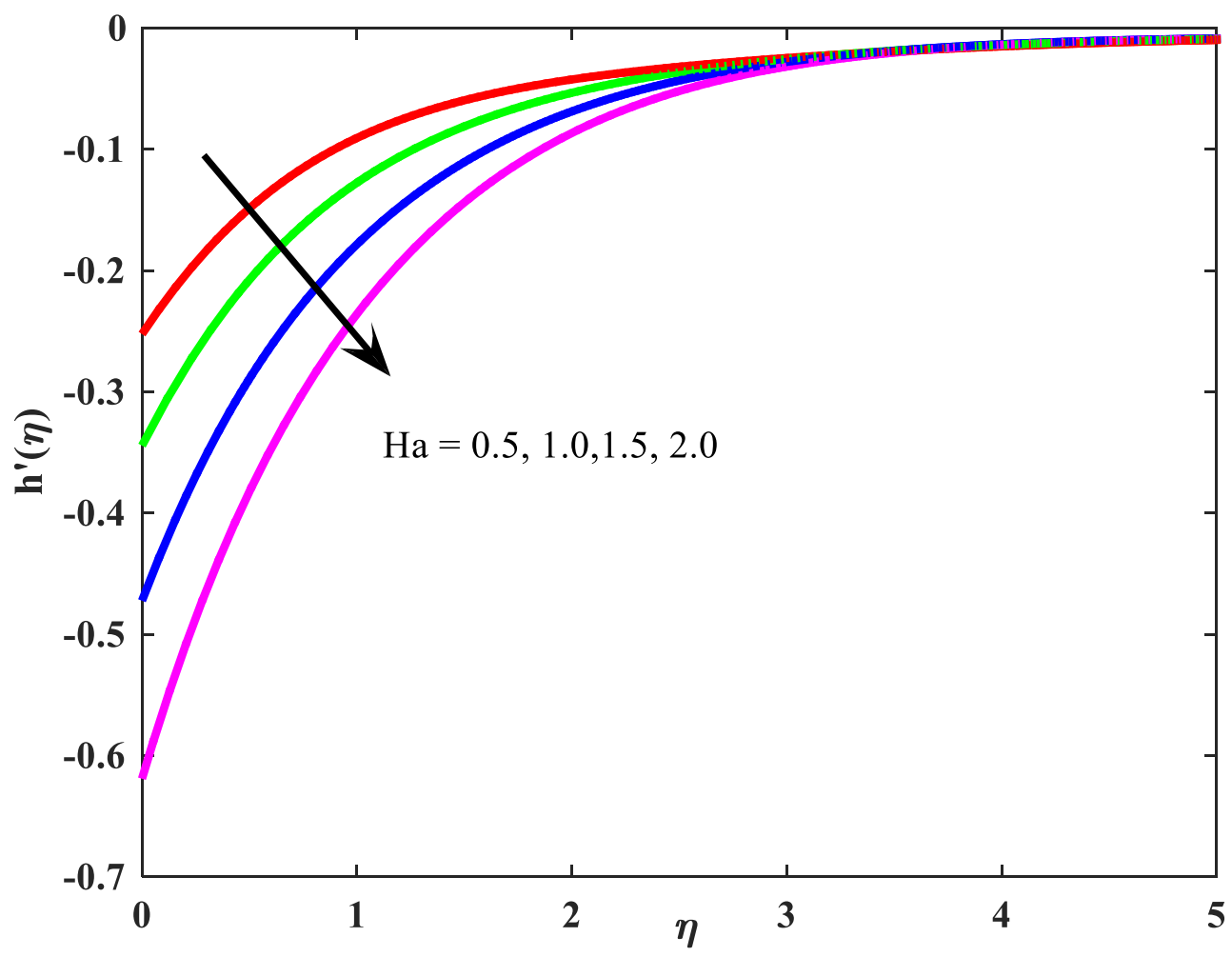

Figure 2(e)-Variations of $H a$ on $h^{\prime}(\eta)$

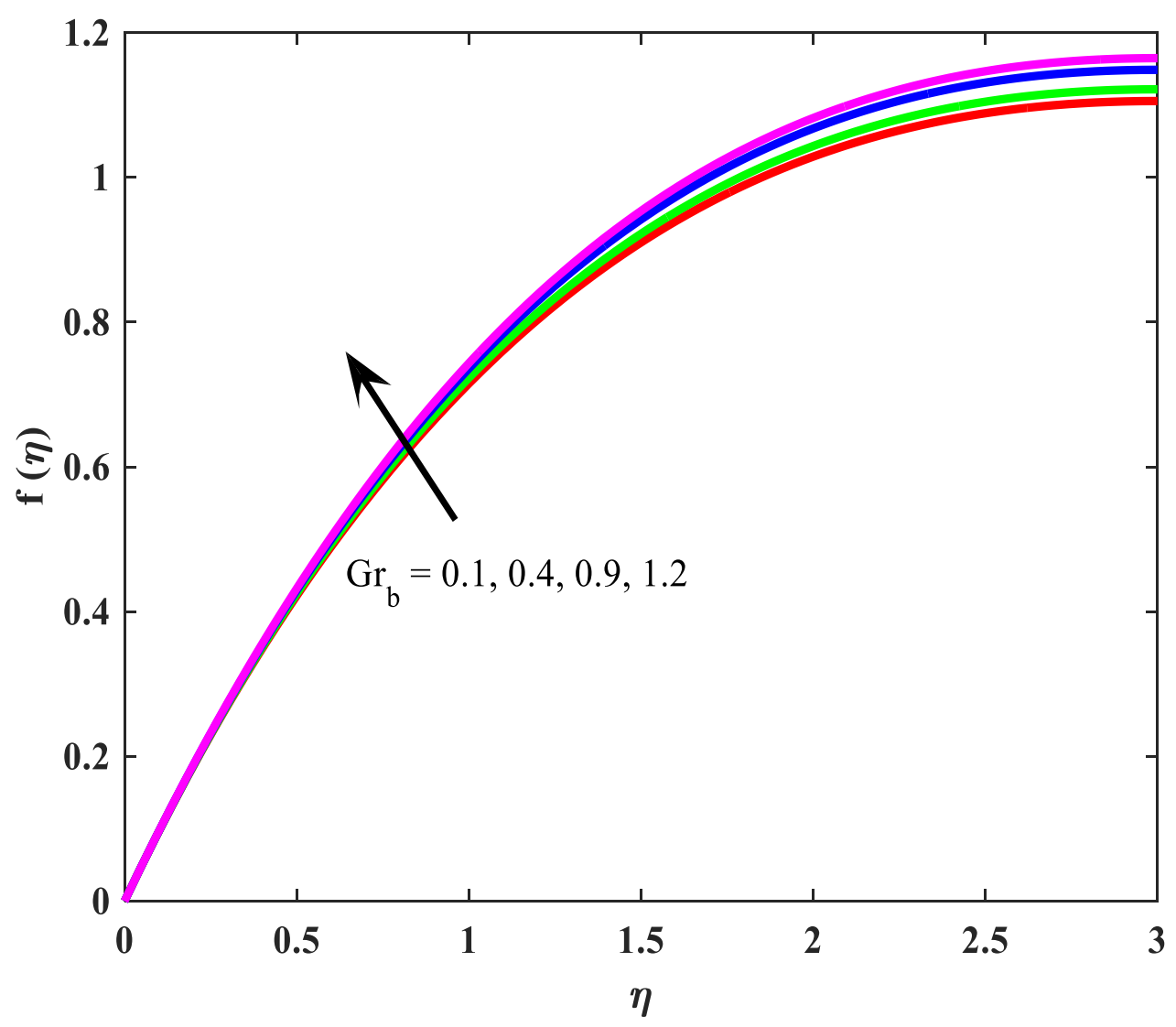

Figure 3(a)-Variations of $G r_{b}$ on $f(\eta)$ 


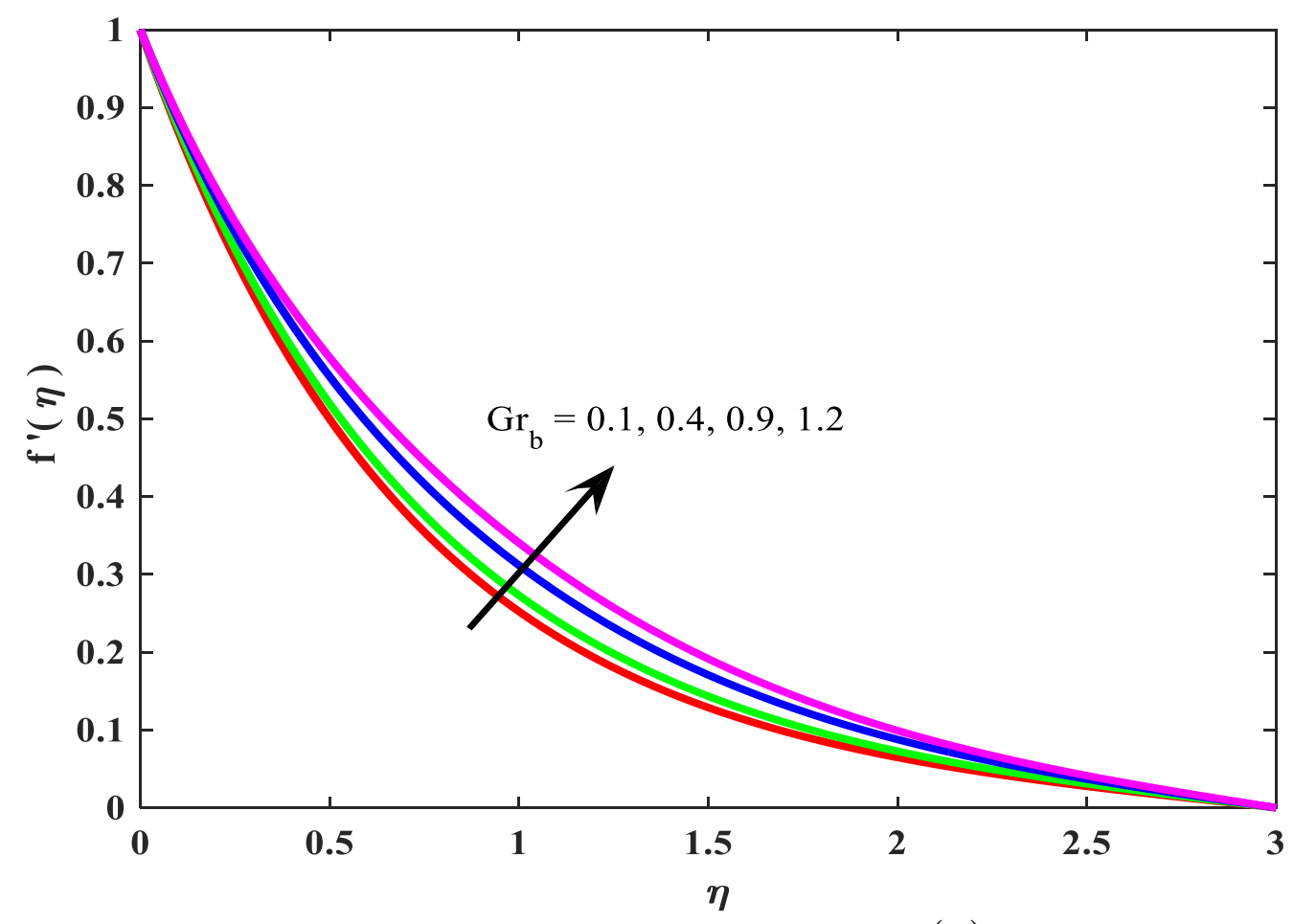

Figure 3(b)-Variations of $G r_{b}$ on $f^{\prime}(\eta)$

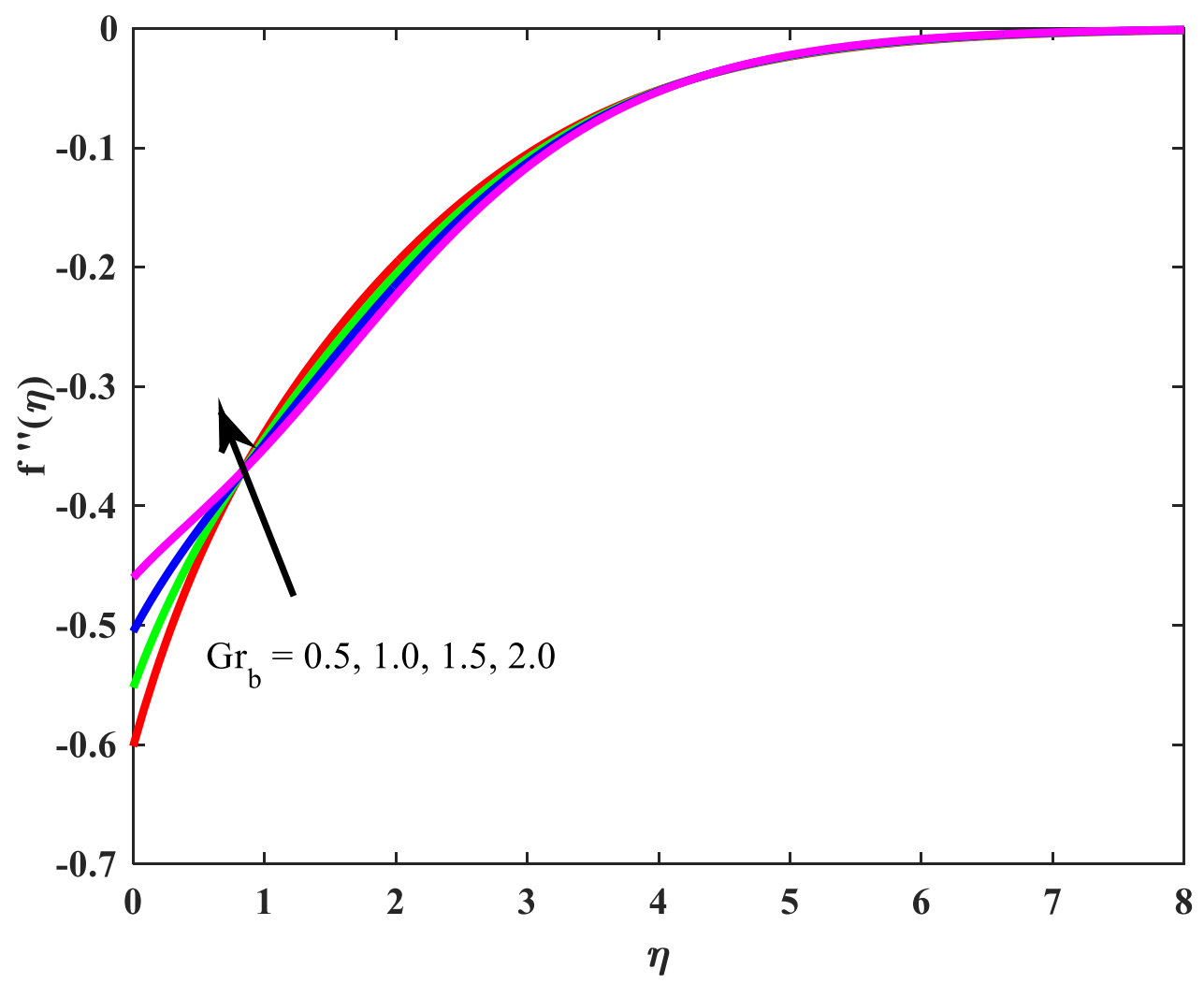

Figure 3(c)-Variations of $G r_{b}$ on $f^{\prime \prime}(\eta)$ 


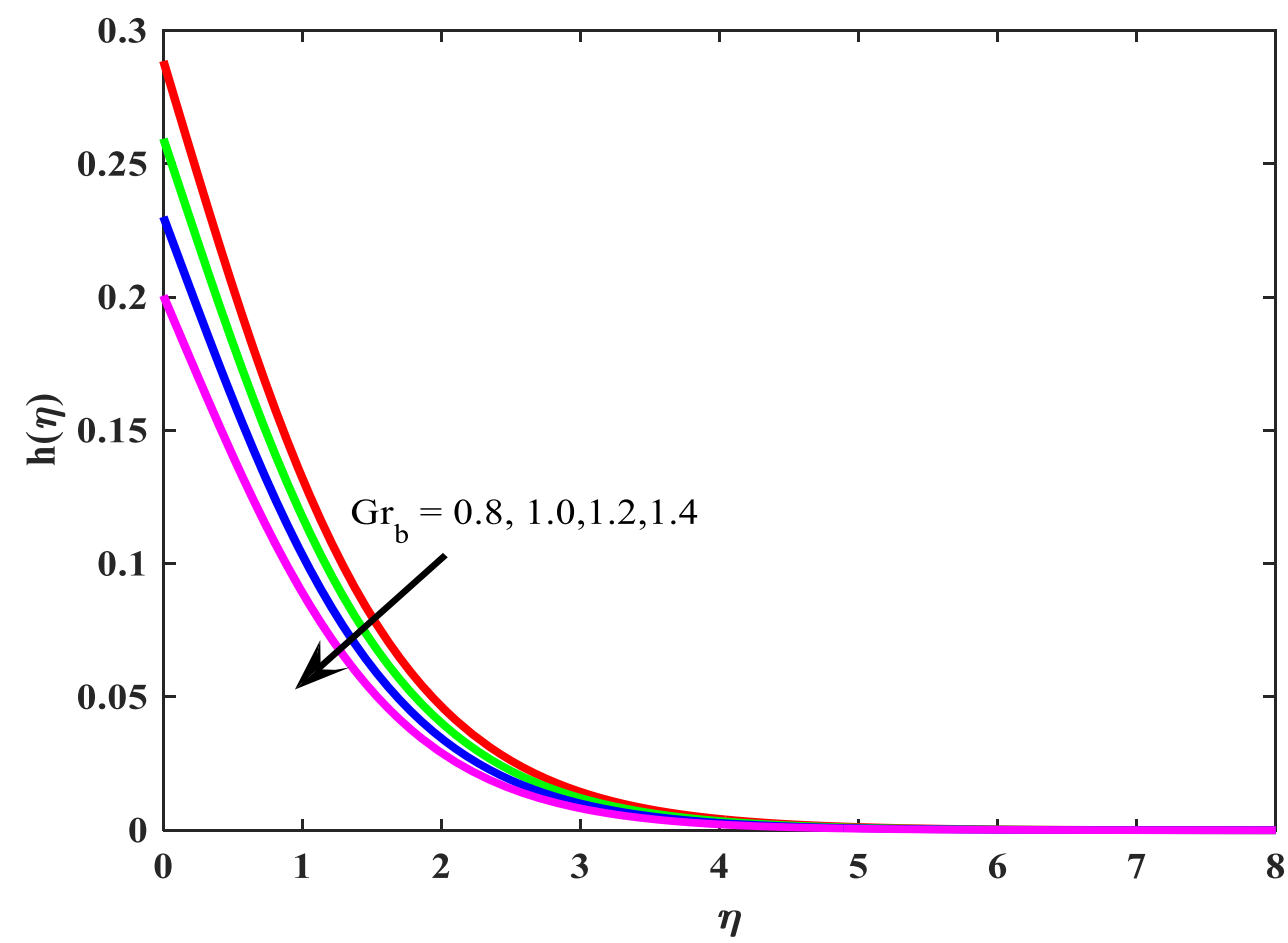

Figure 3(d)-Variations of $G r_{b}$ on $h(\eta)$

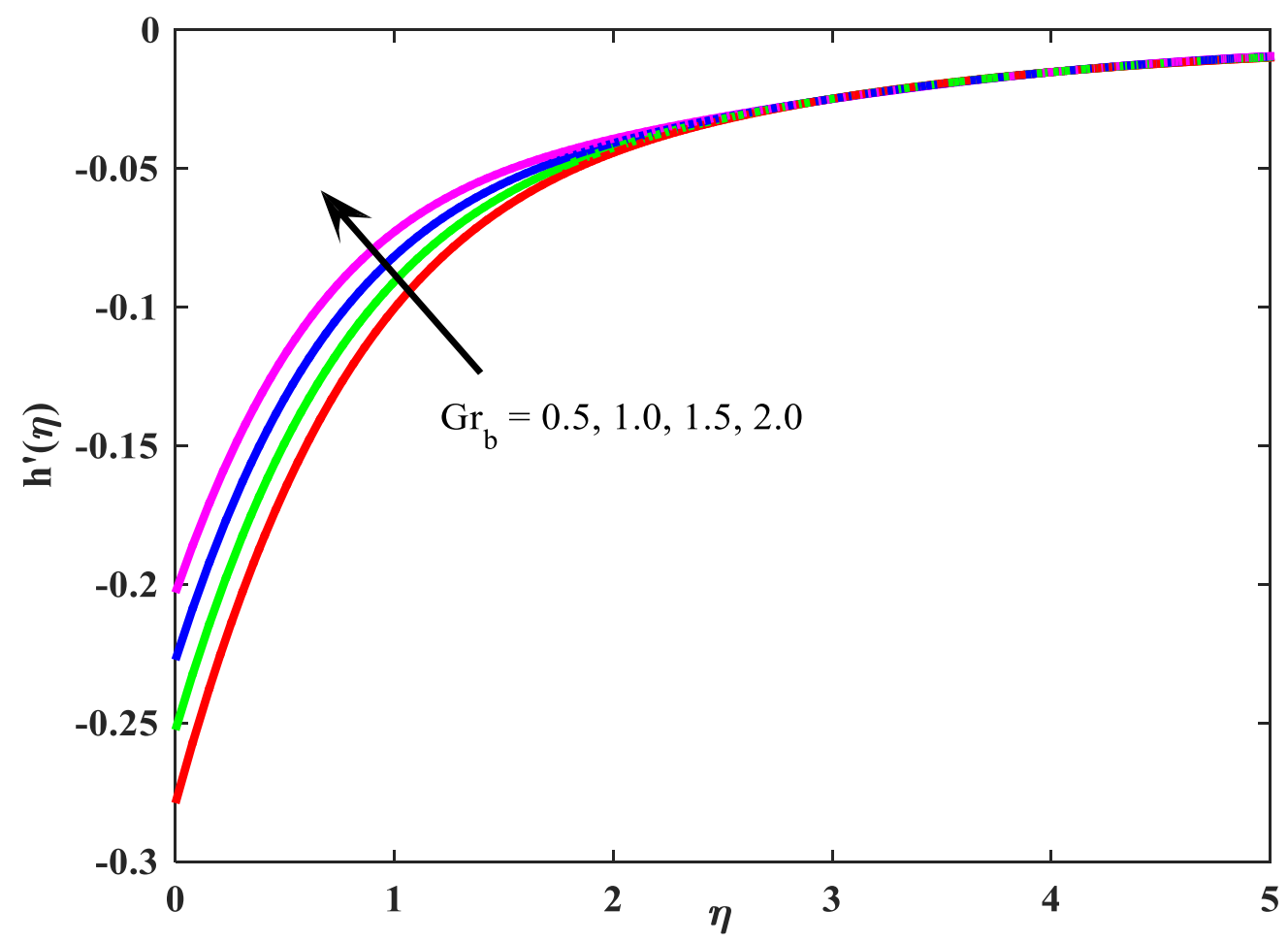

Figure 3(e)-Variations of $G r_{b}$ on $h^{\prime}(\eta)$ 


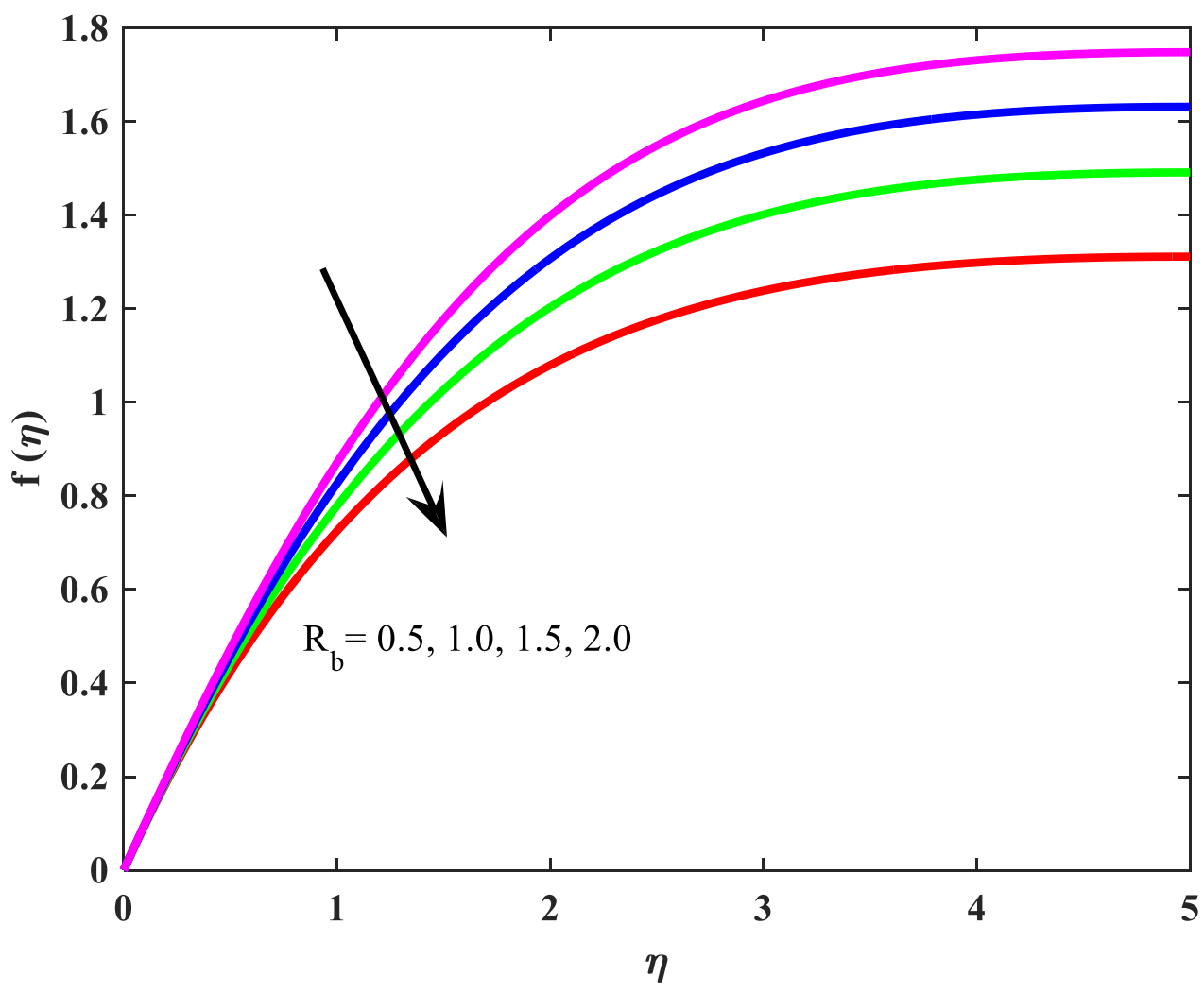

Figure 4(a)-Variations of $R_{b}$ on $f(\eta)$

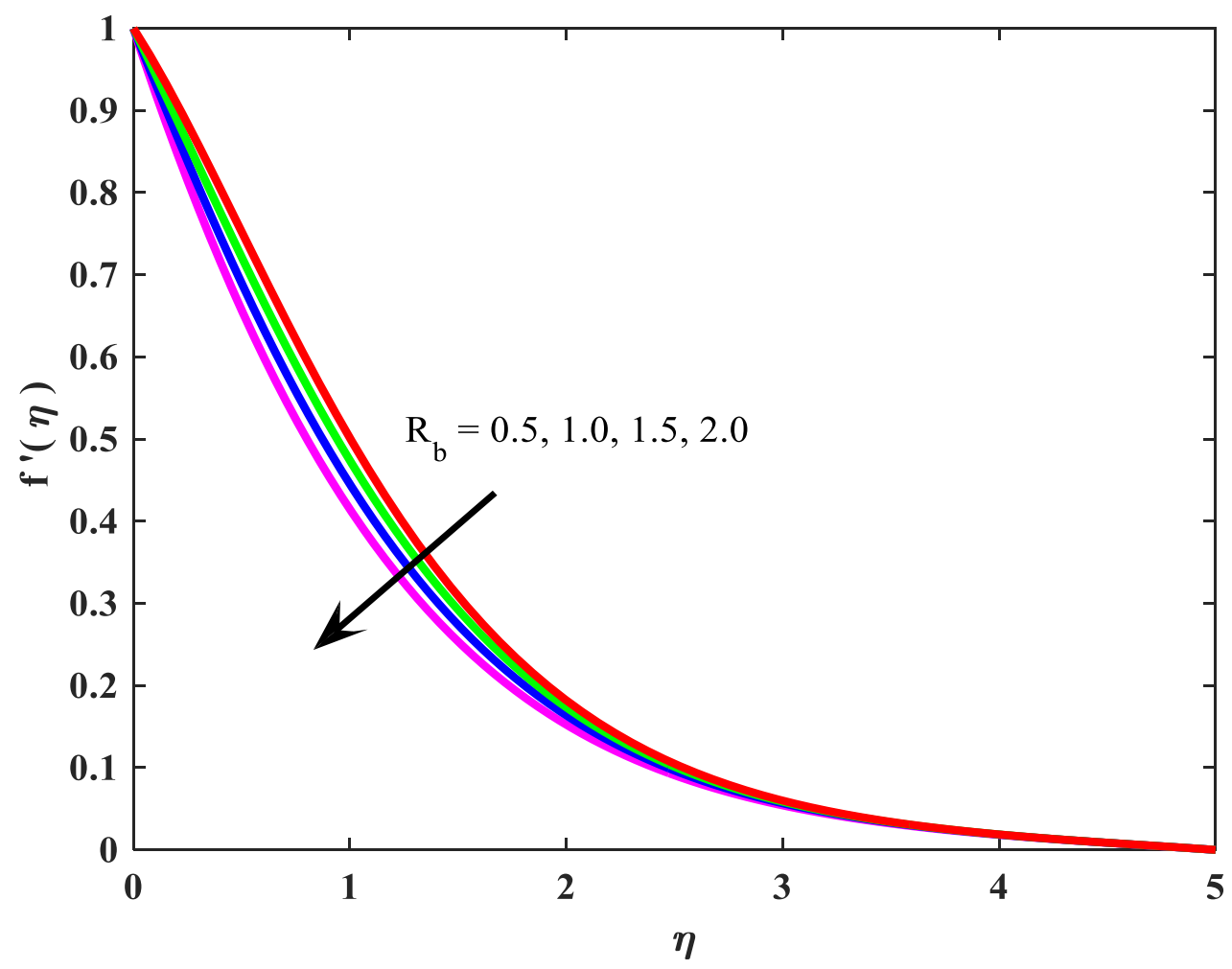

Figure 4(b)-Variations of $R_{b}$ on $f^{\prime}(\eta)$ 


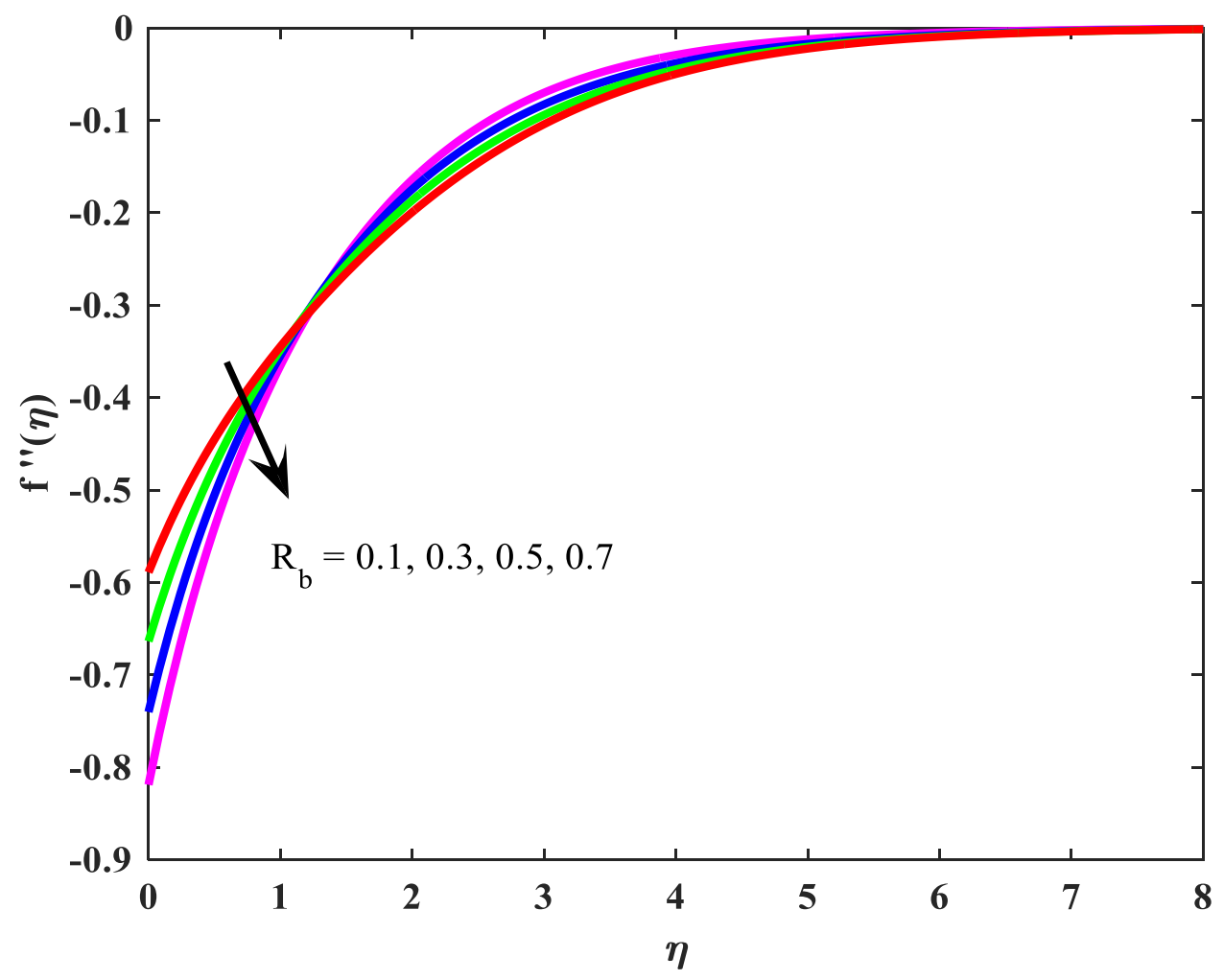

Figure 4(c)-Variations of $R_{b}$ on $f^{\prime \prime}(\eta)$

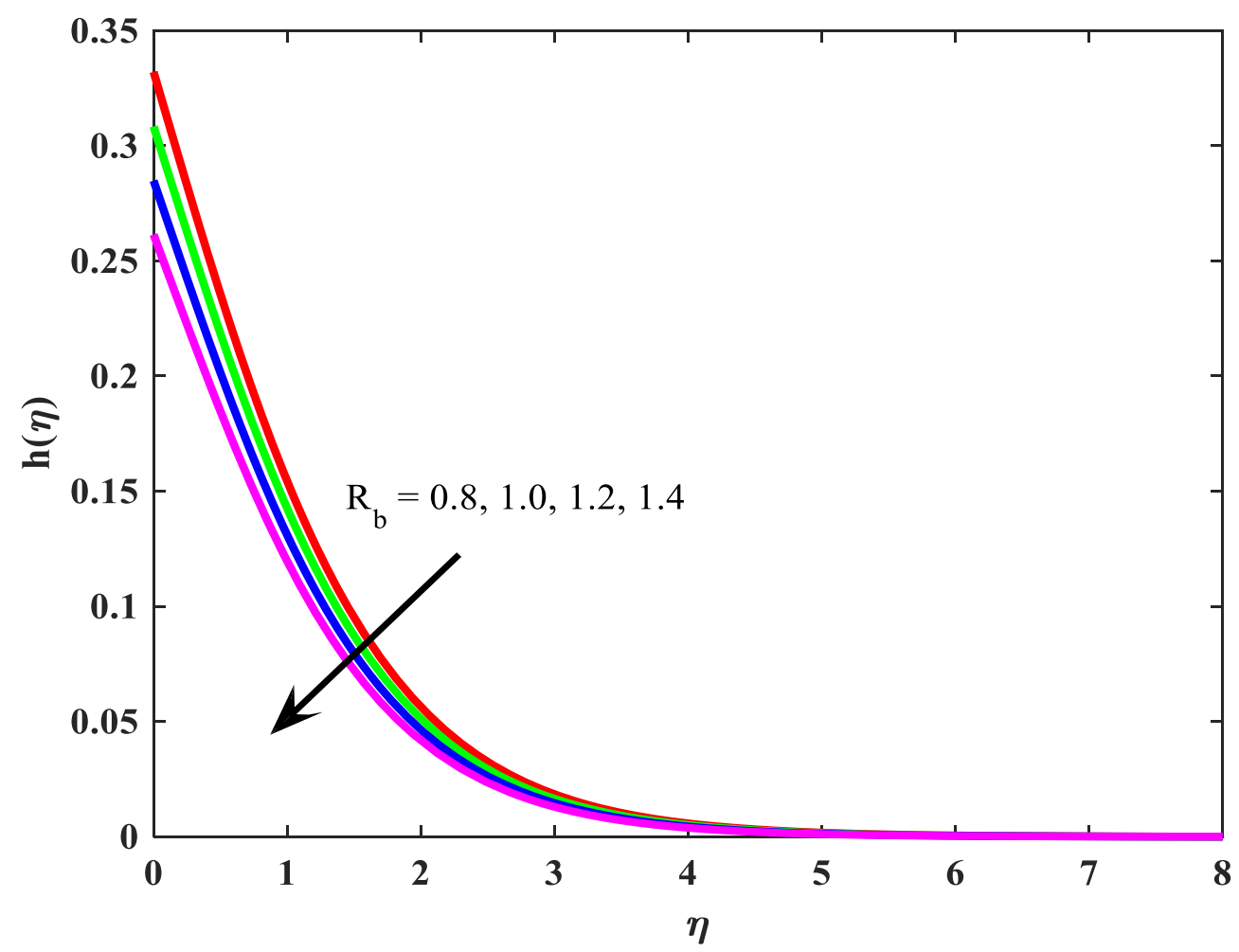

Figure 4(d)-Variations of $R_{b}$ on $h(\eta)$ 


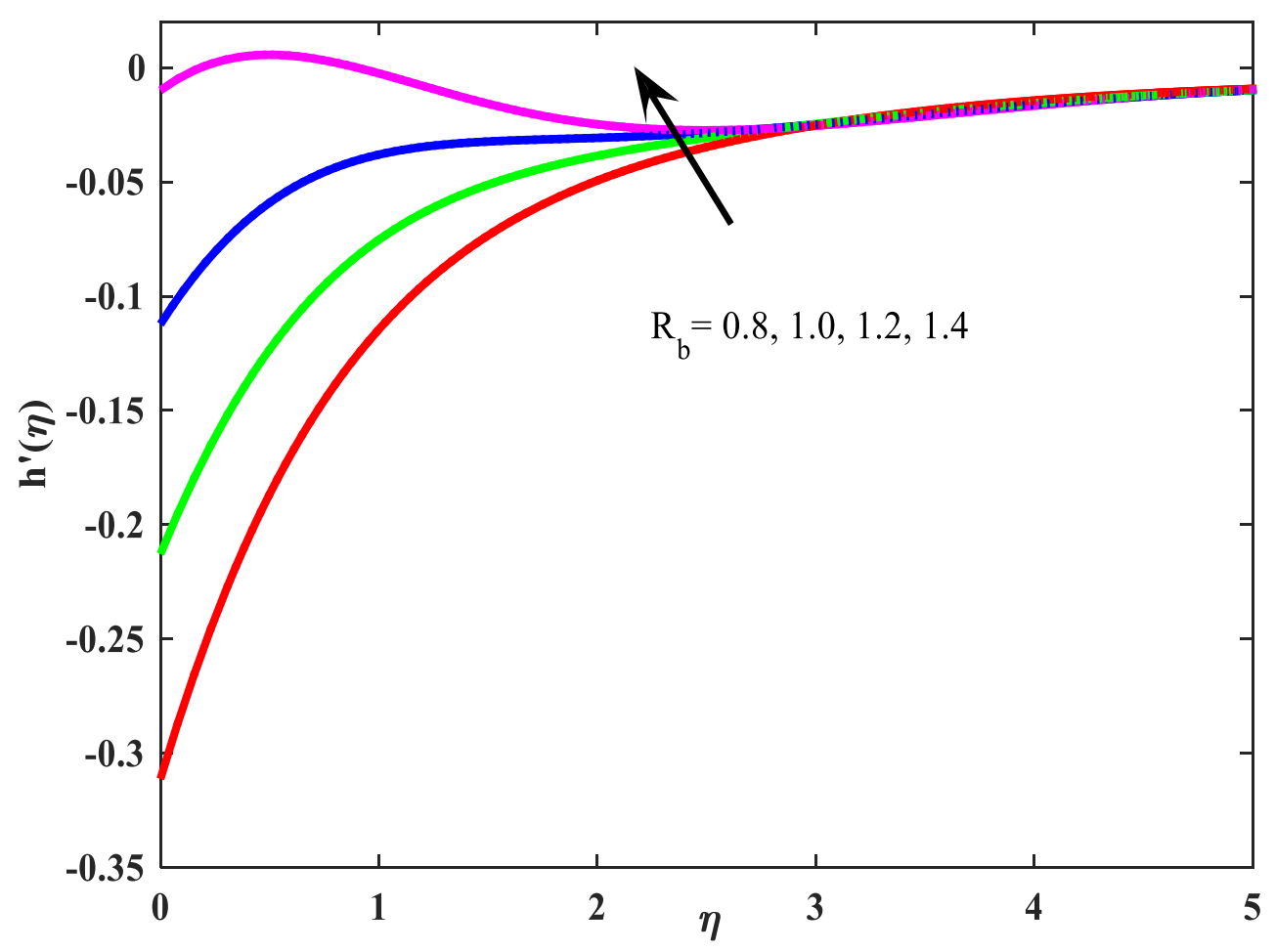

Figure 4(e)-Variations of $R_{b}$ on $h^{\prime}(\eta)$

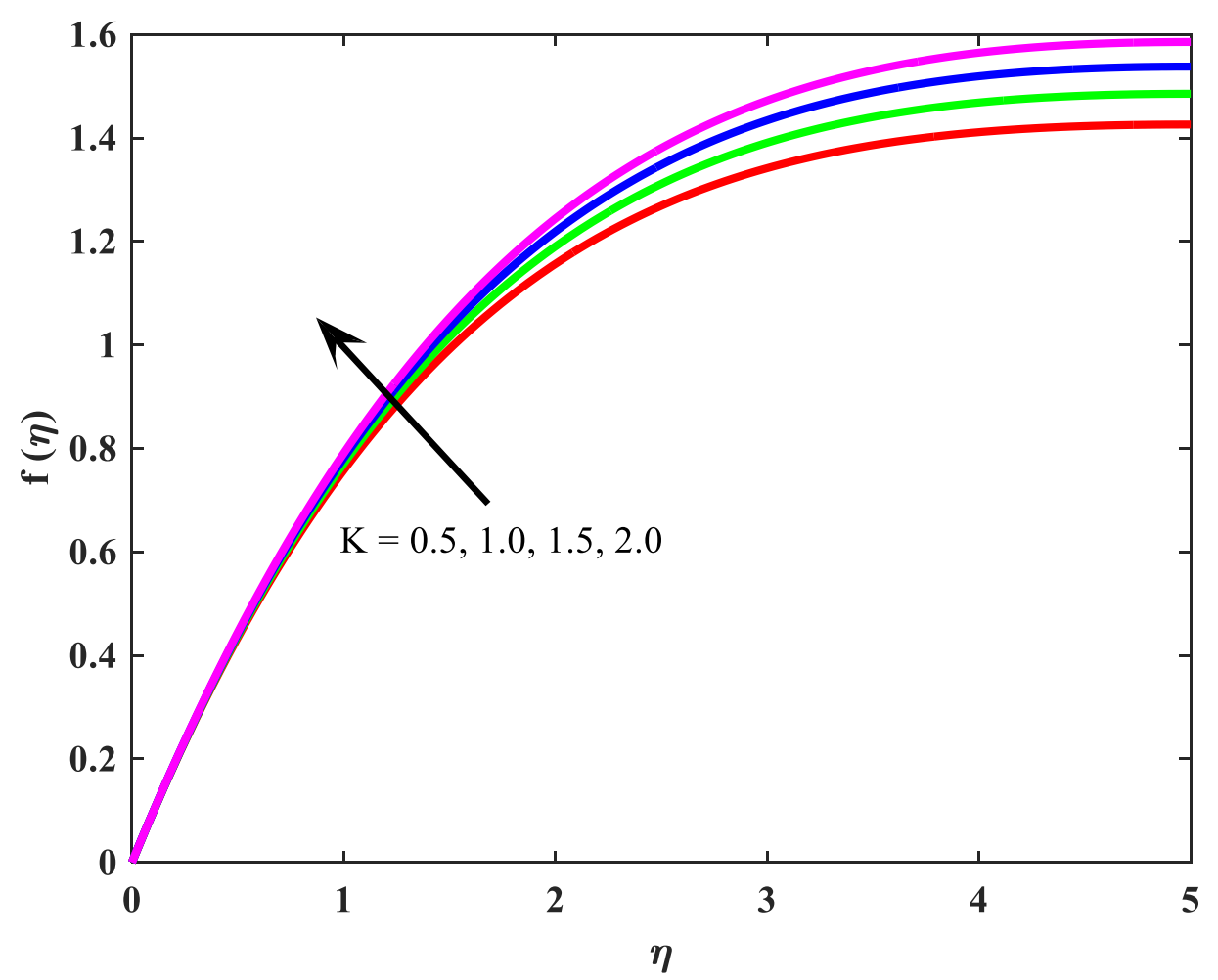

Figure 5(a)-Variations of $K$ on $f(\eta)$ 


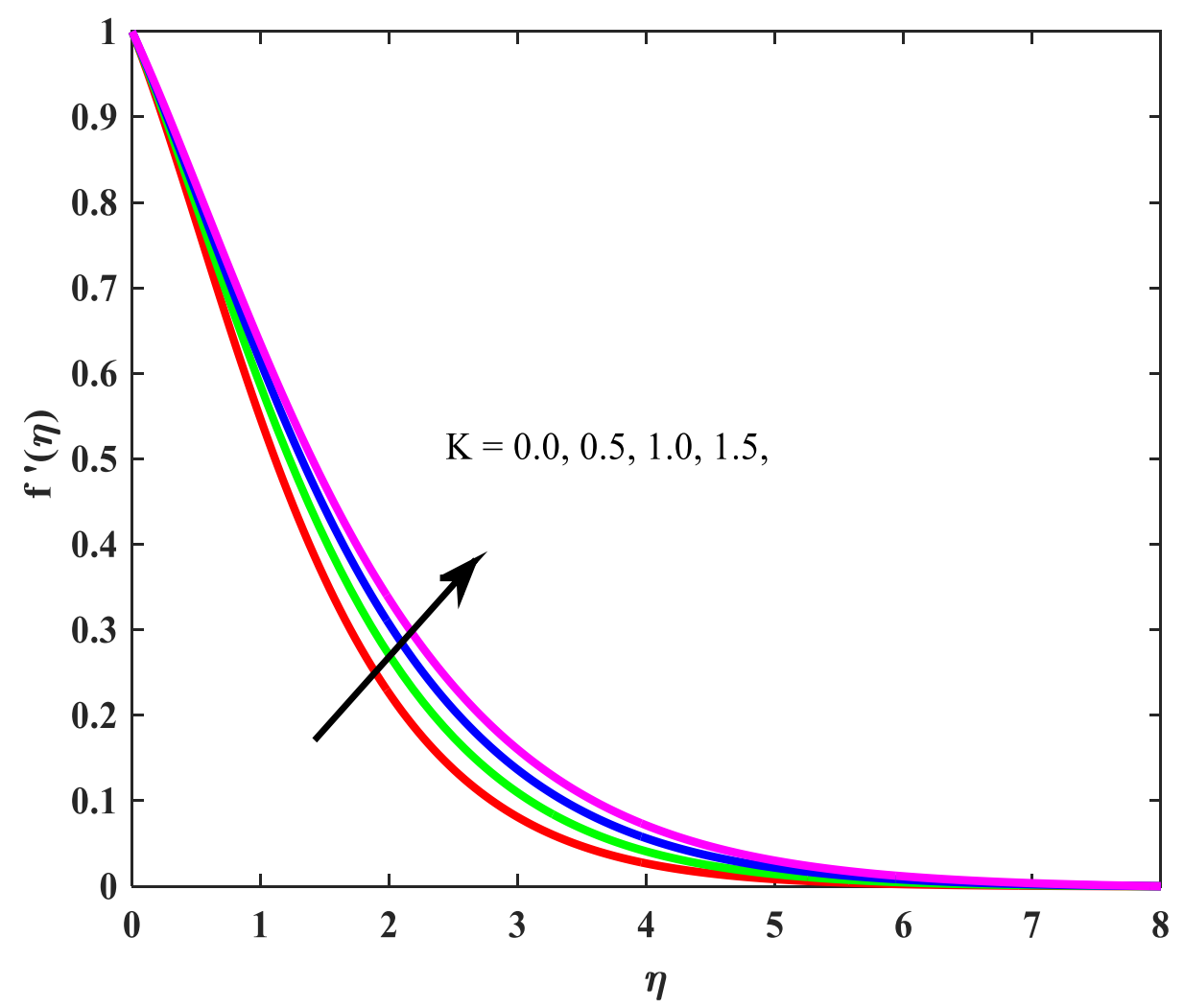

Figure 5(b)-Variations of $K$ on $f^{\prime}(\eta)$

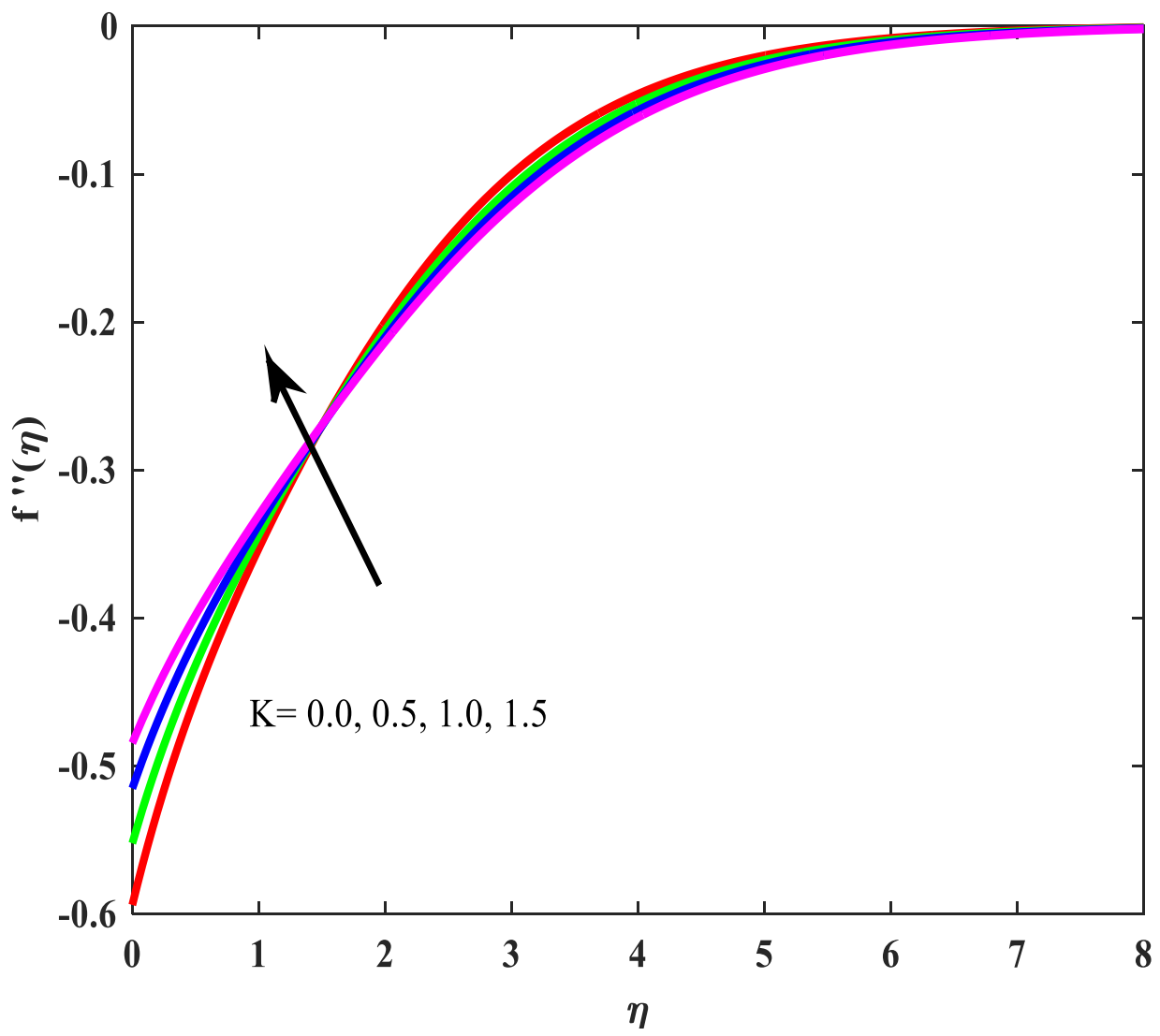

Figure 5(c)-Variations of $K$ on $f^{\prime \prime}(\eta)$ 


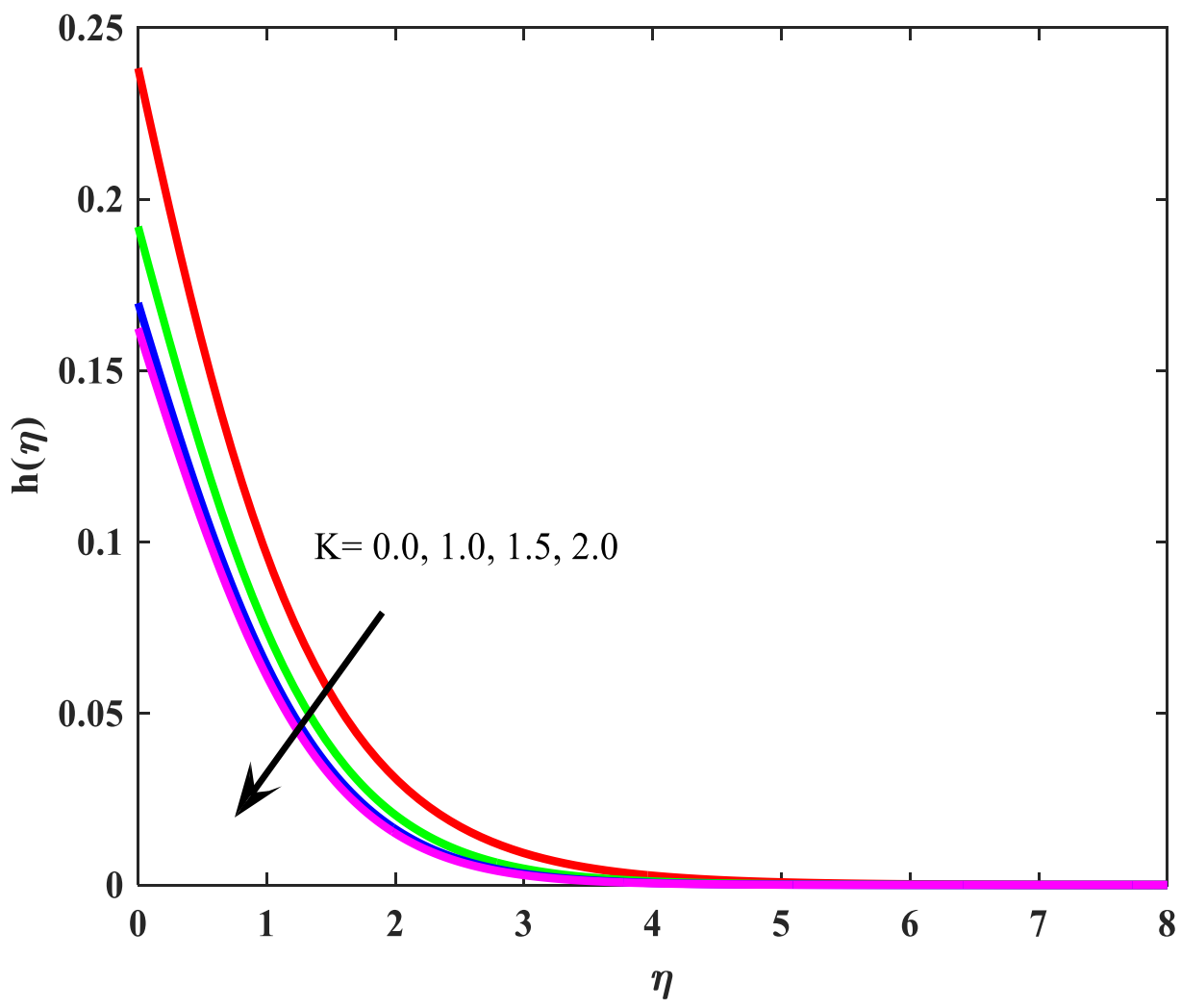

Figure 5(d)-Variations of $K$ on $h(\eta)$

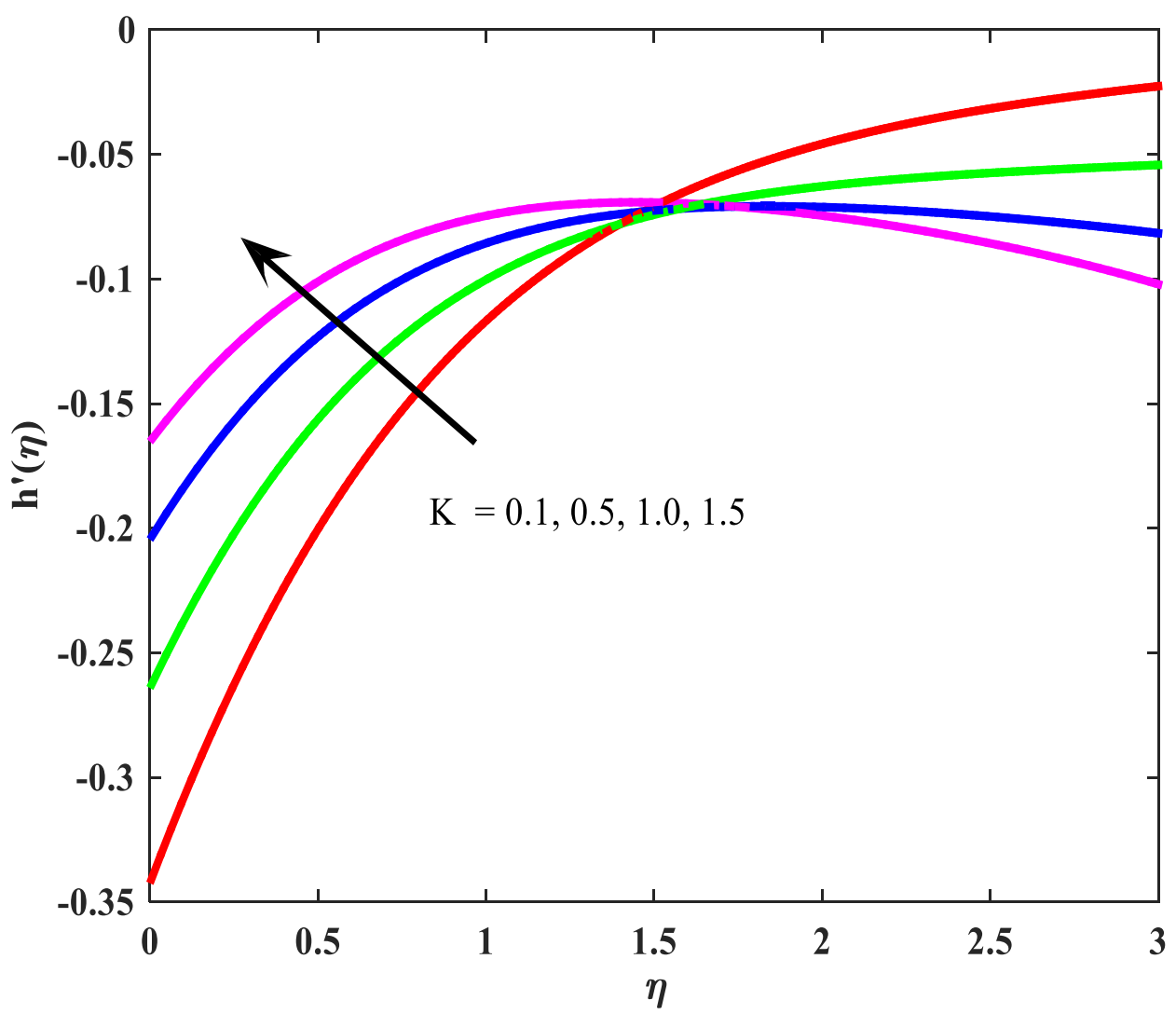

Figure 5(e)-Variations of $K$ on $h^{\prime}(\eta)$ 


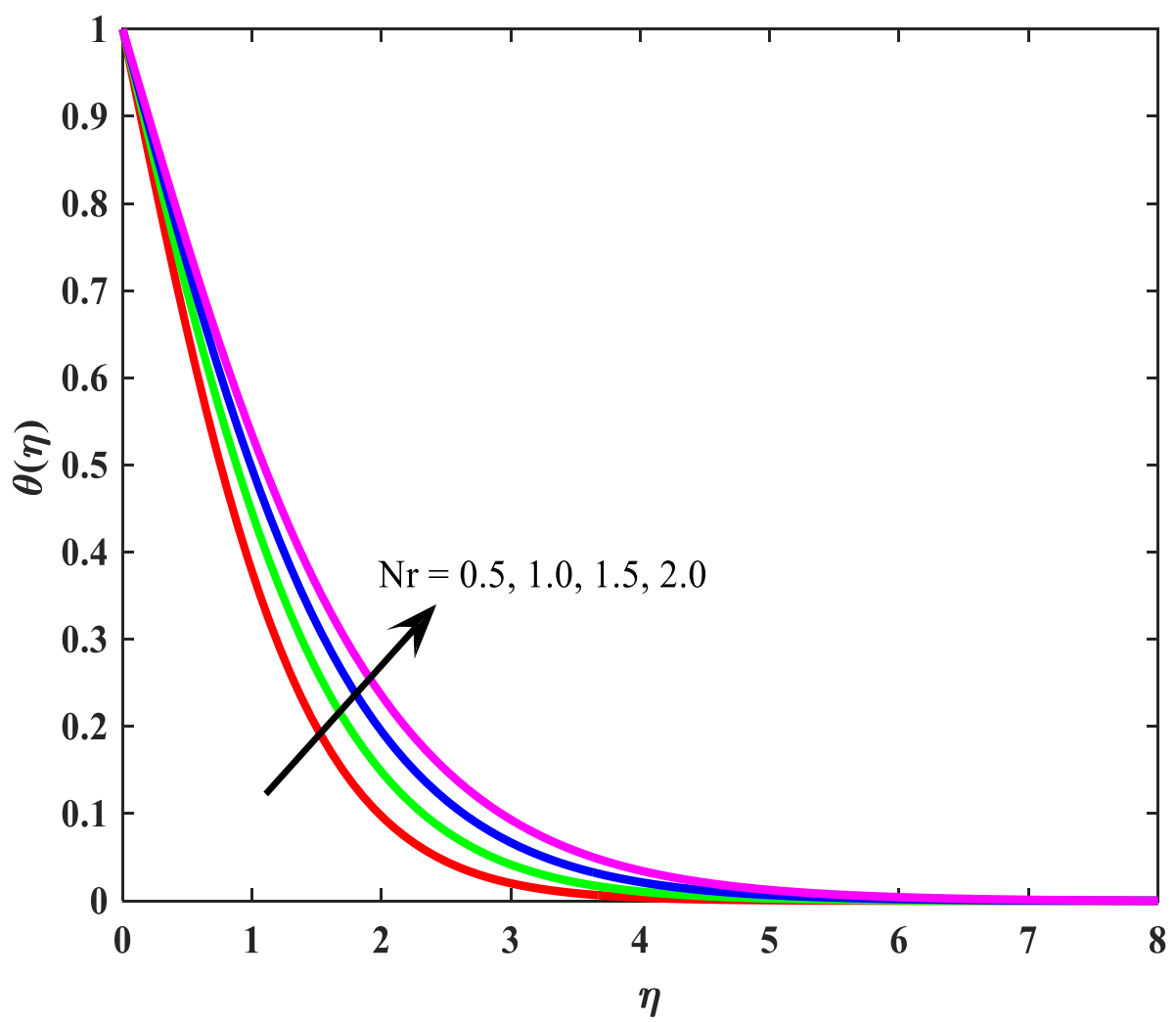

Figure 6(a)-Variations of $N r$ on $\theta(\eta)$

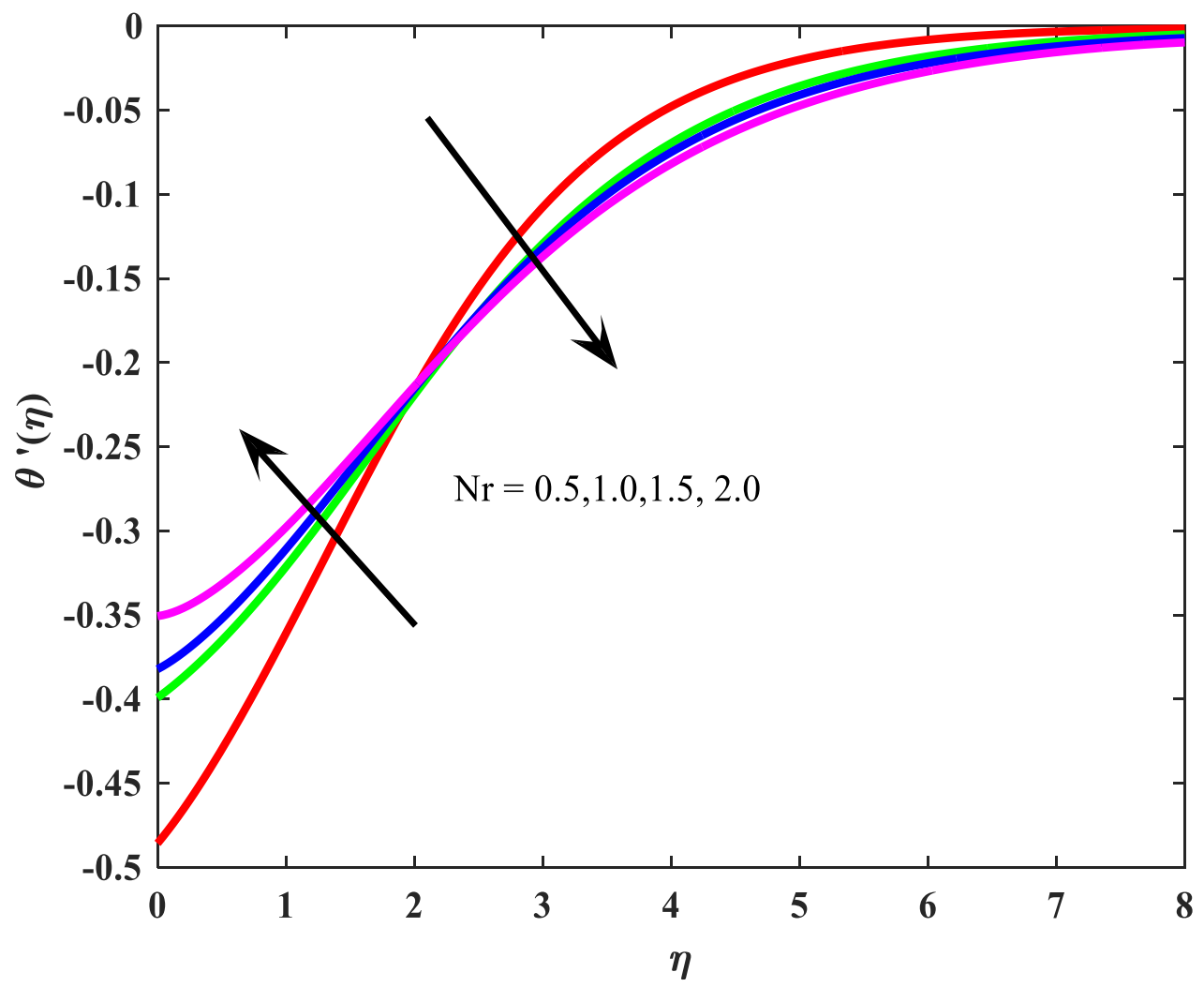

Figure 6(b)-Variations of $N r$ on $\theta^{\prime}(\eta)$ 


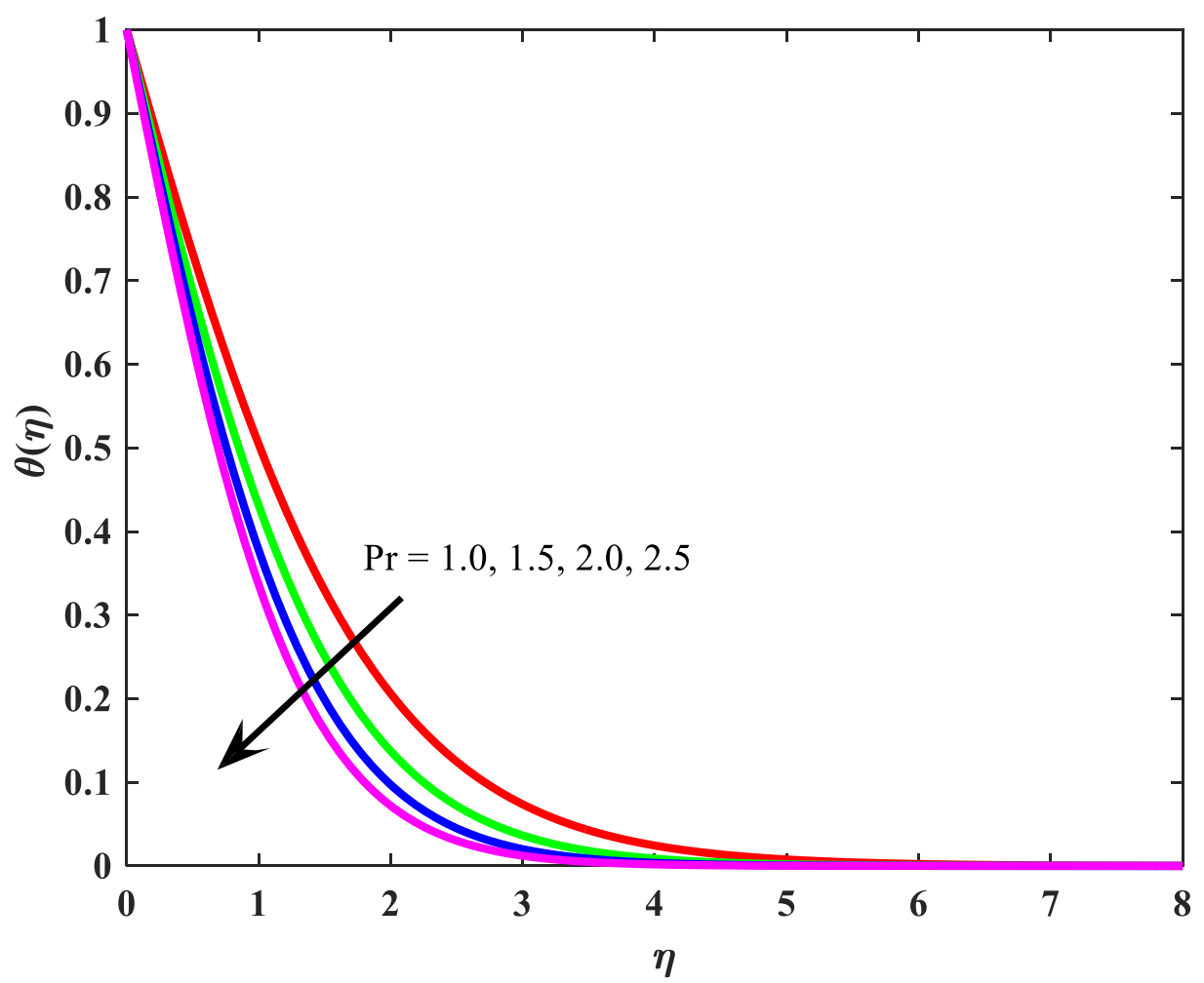

Figure 7(a)-Variations of $\operatorname{Pr}$ on $\theta(\eta)$

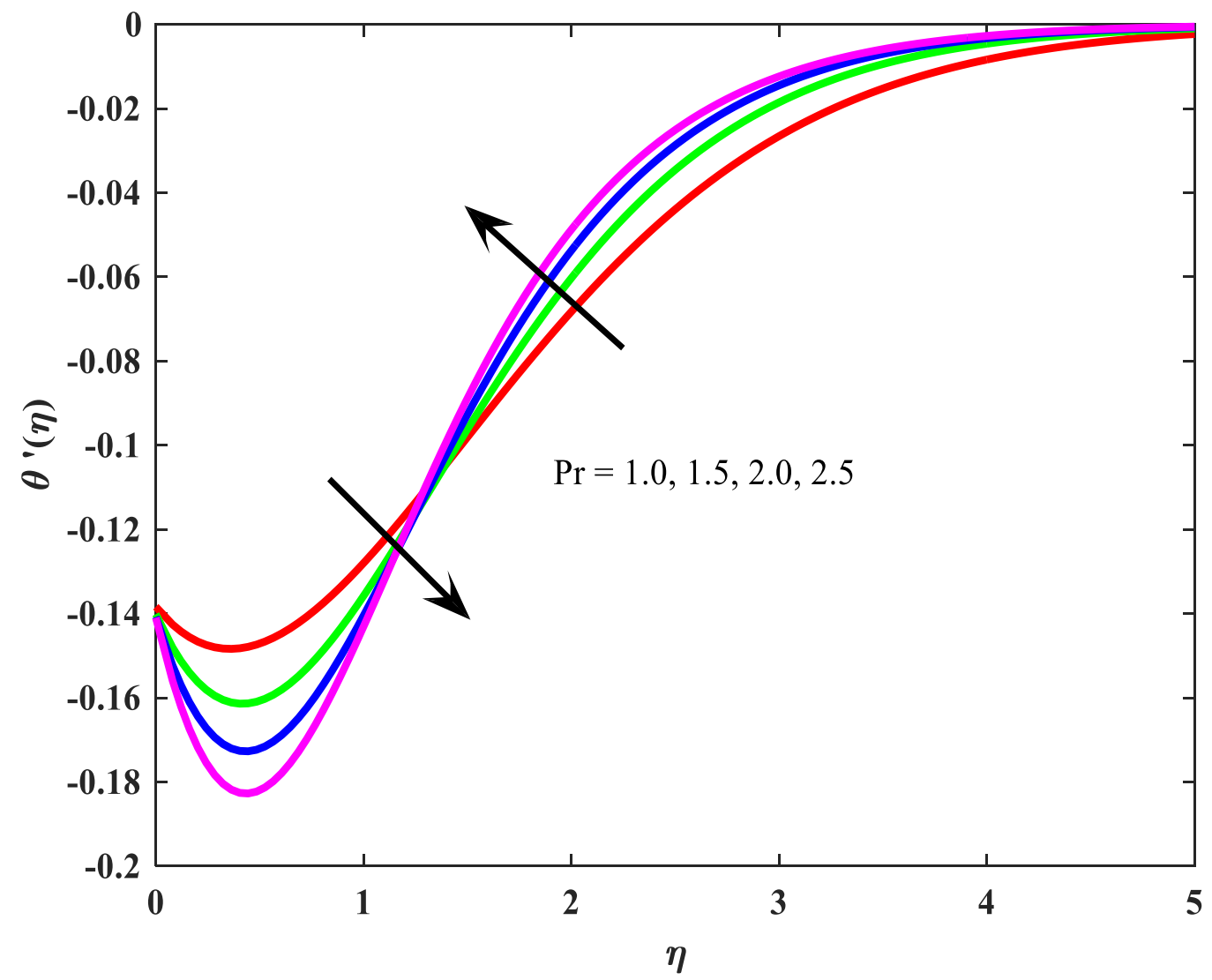

Figure 7(b)-Variations of $\operatorname{Pr}$ on $\theta^{\prime}(\eta)$ 


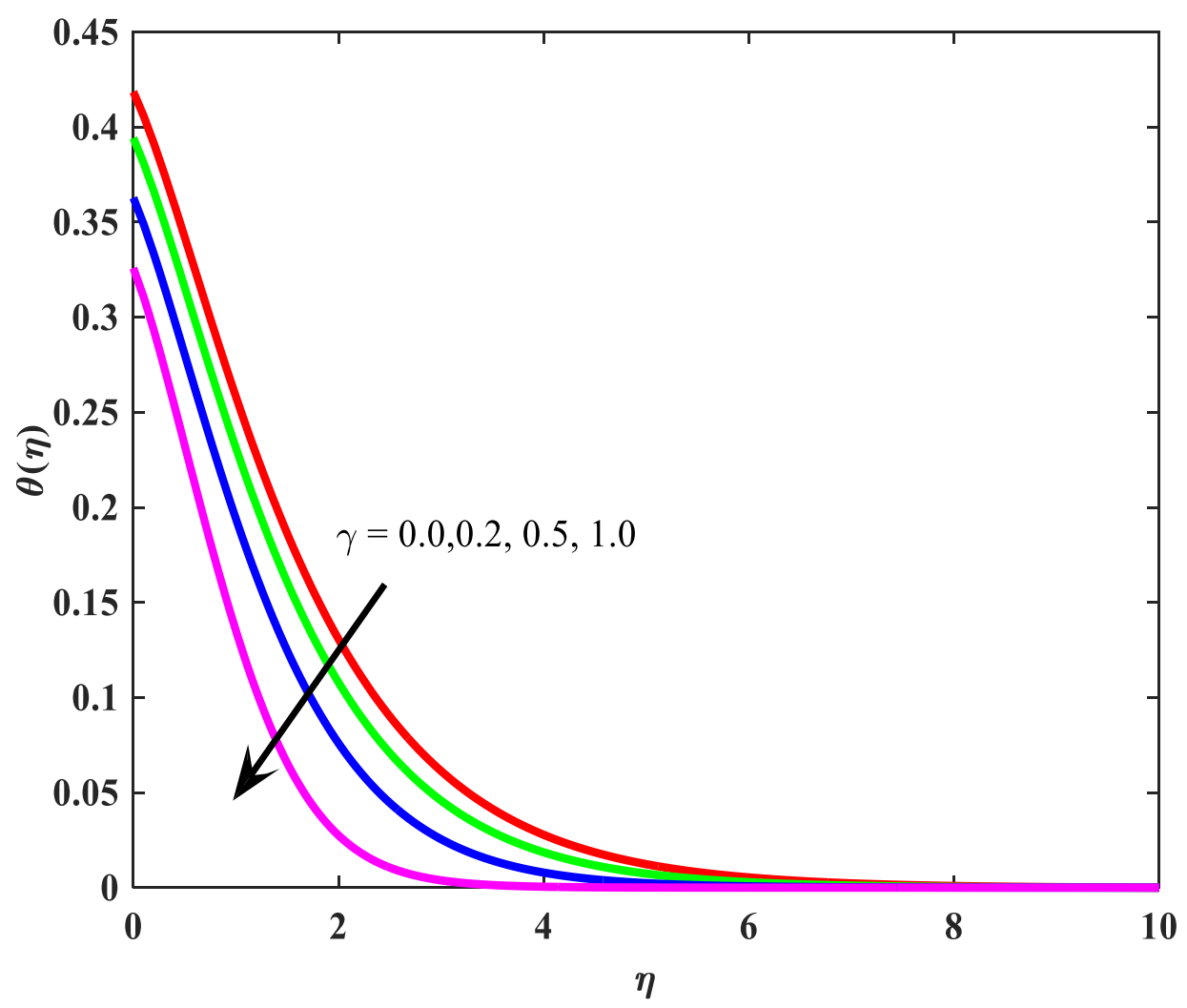

Figure 8(a)-Variations of $\gamma$ on $\theta(\eta)$

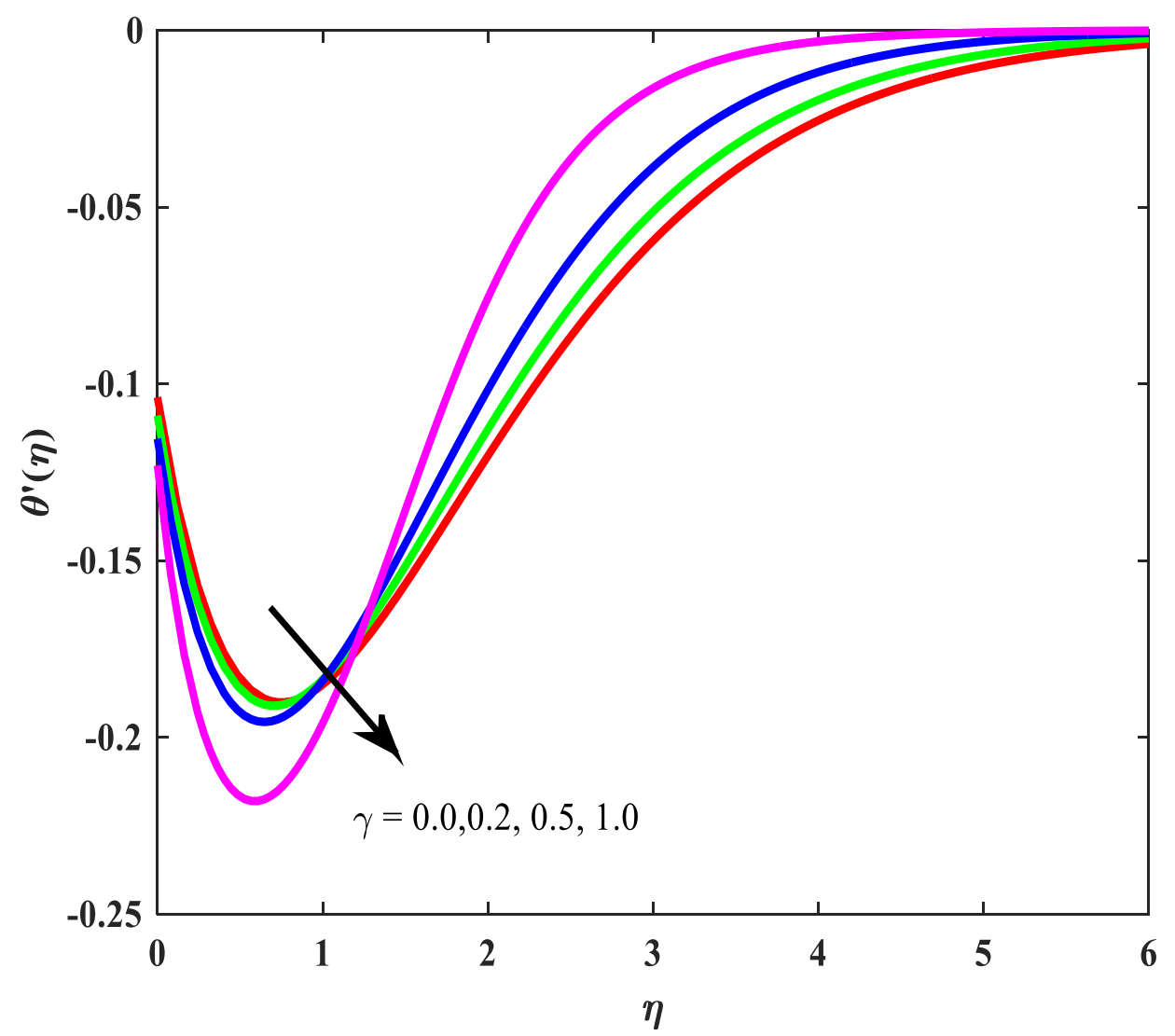

Figure 8(b)-Variations of $\gamma$ on $\theta^{\prime}(\eta)$ 


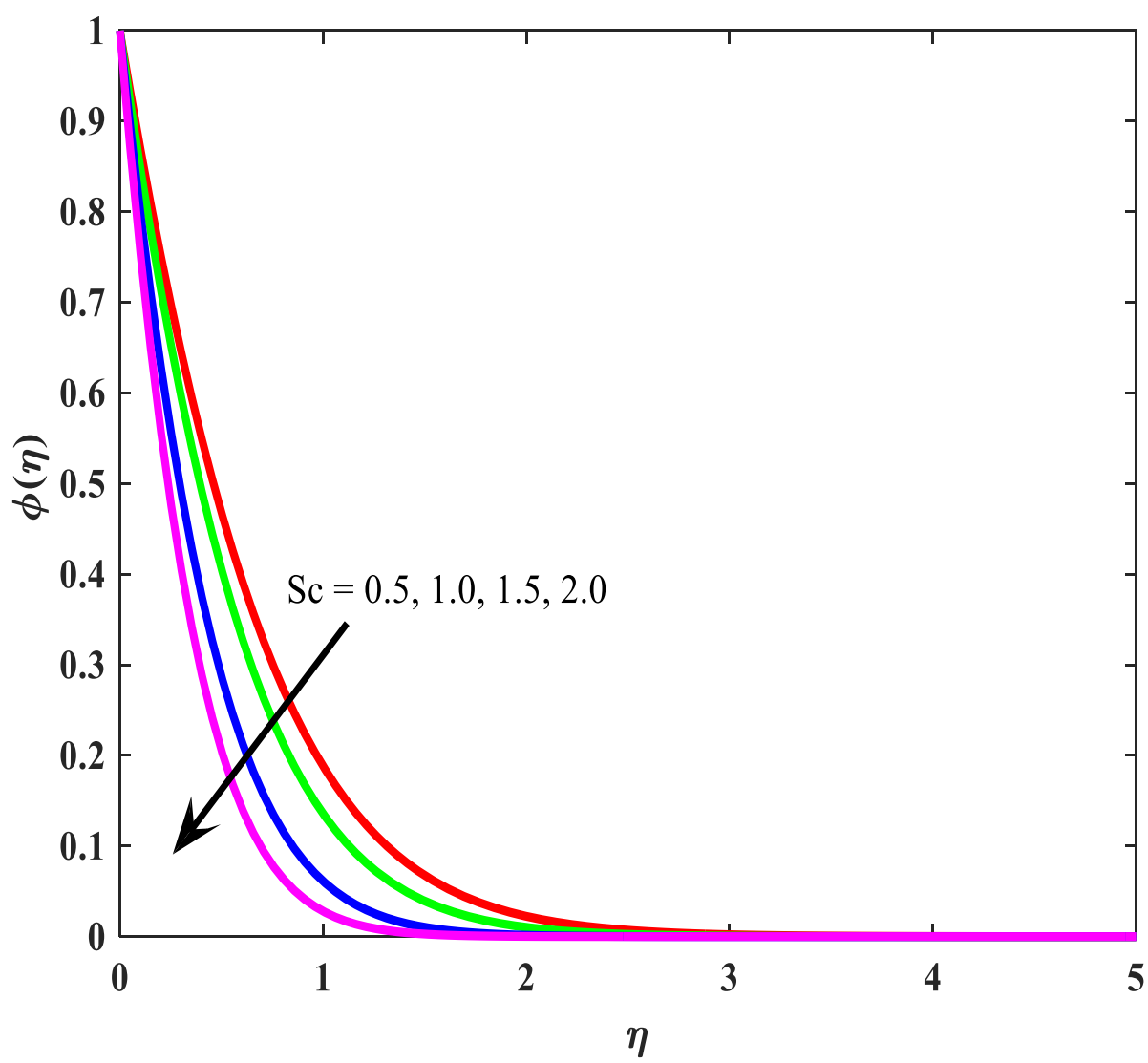

Figure 9(a)-Variations of $S c$ on $\phi(\eta)$

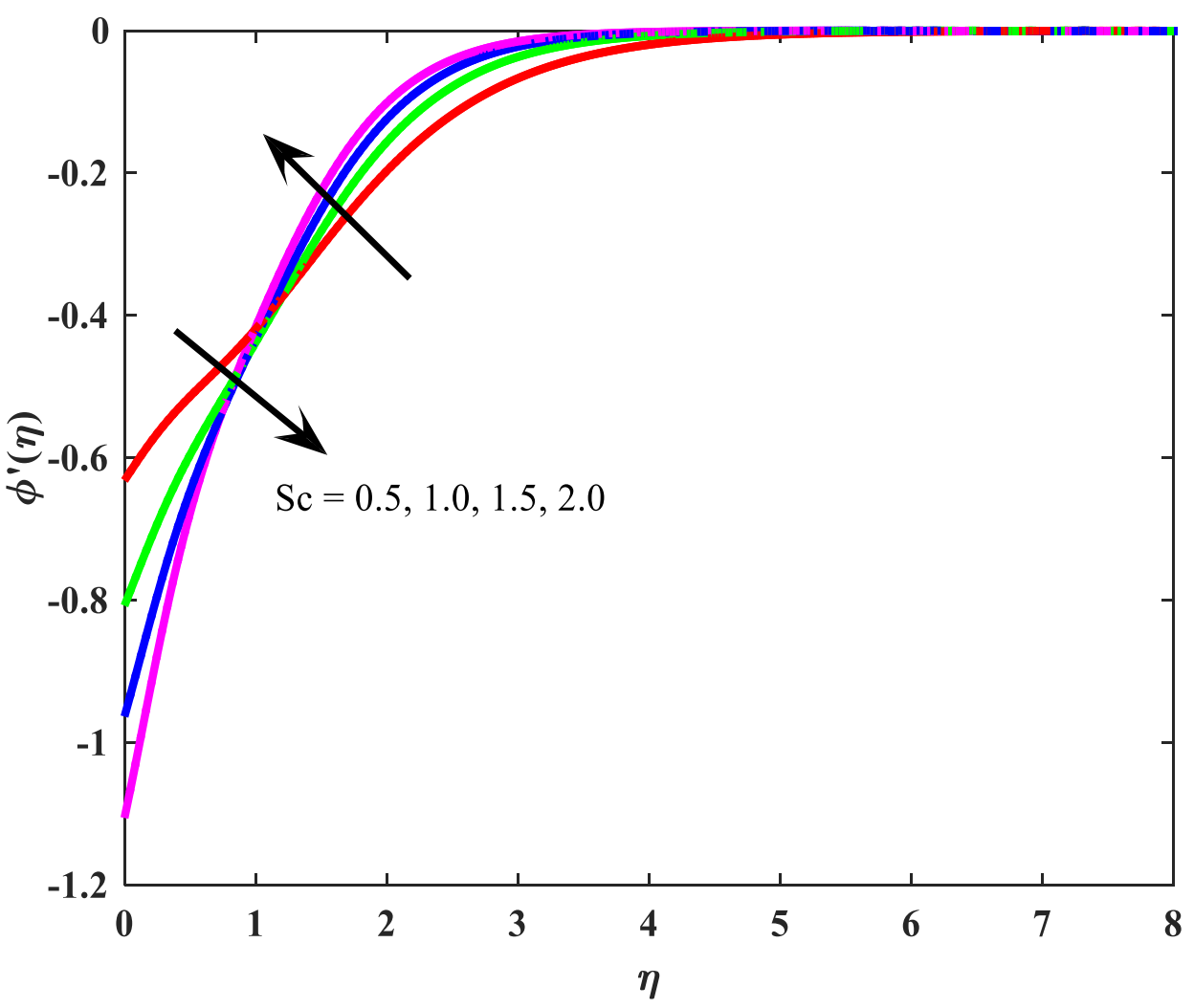

Figure 9(b)-Variations of $S c$ on $\phi^{\prime}(\eta)$ 


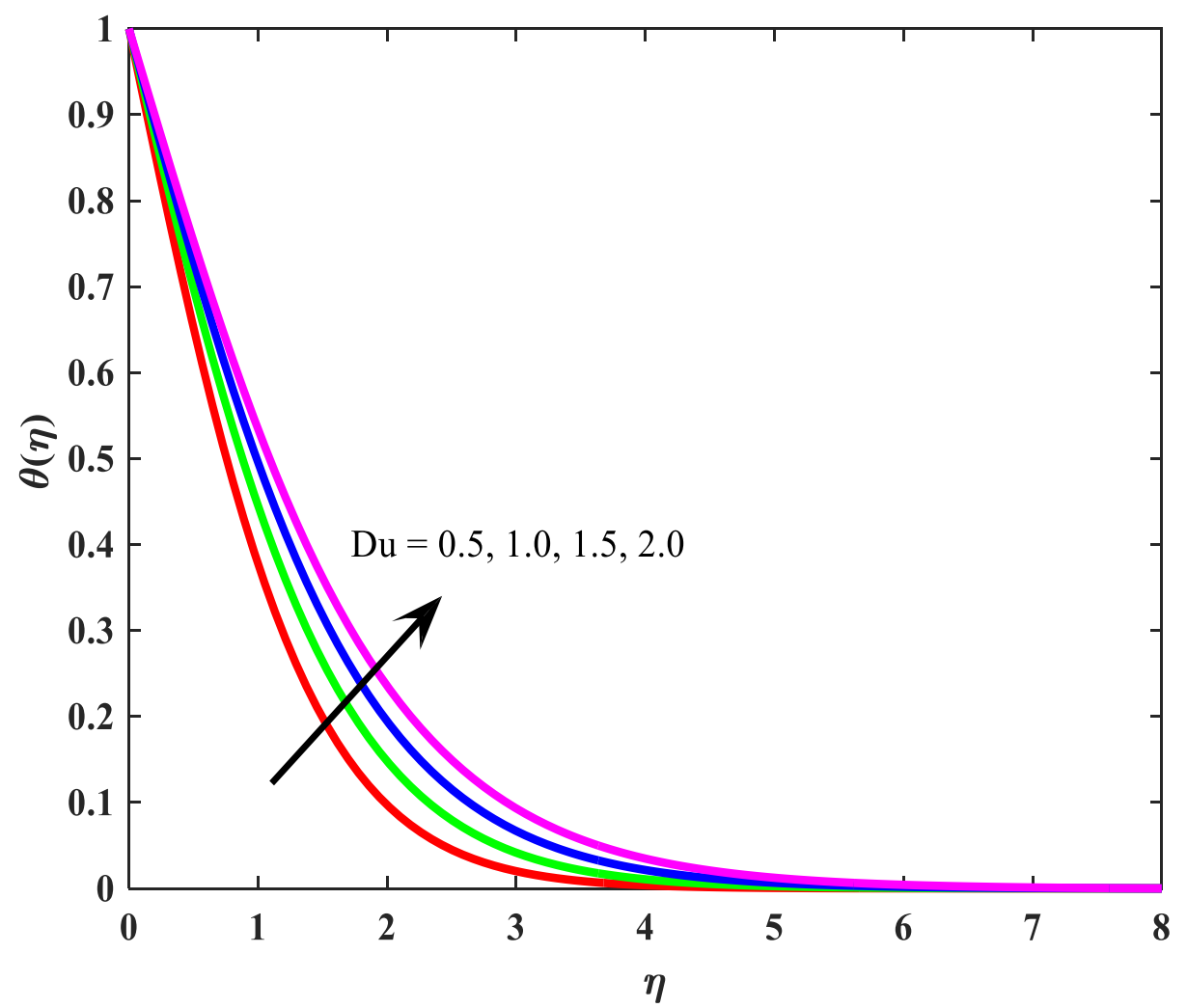

Figure 10(a)-Variations of $D u$ on $\theta(\eta)$

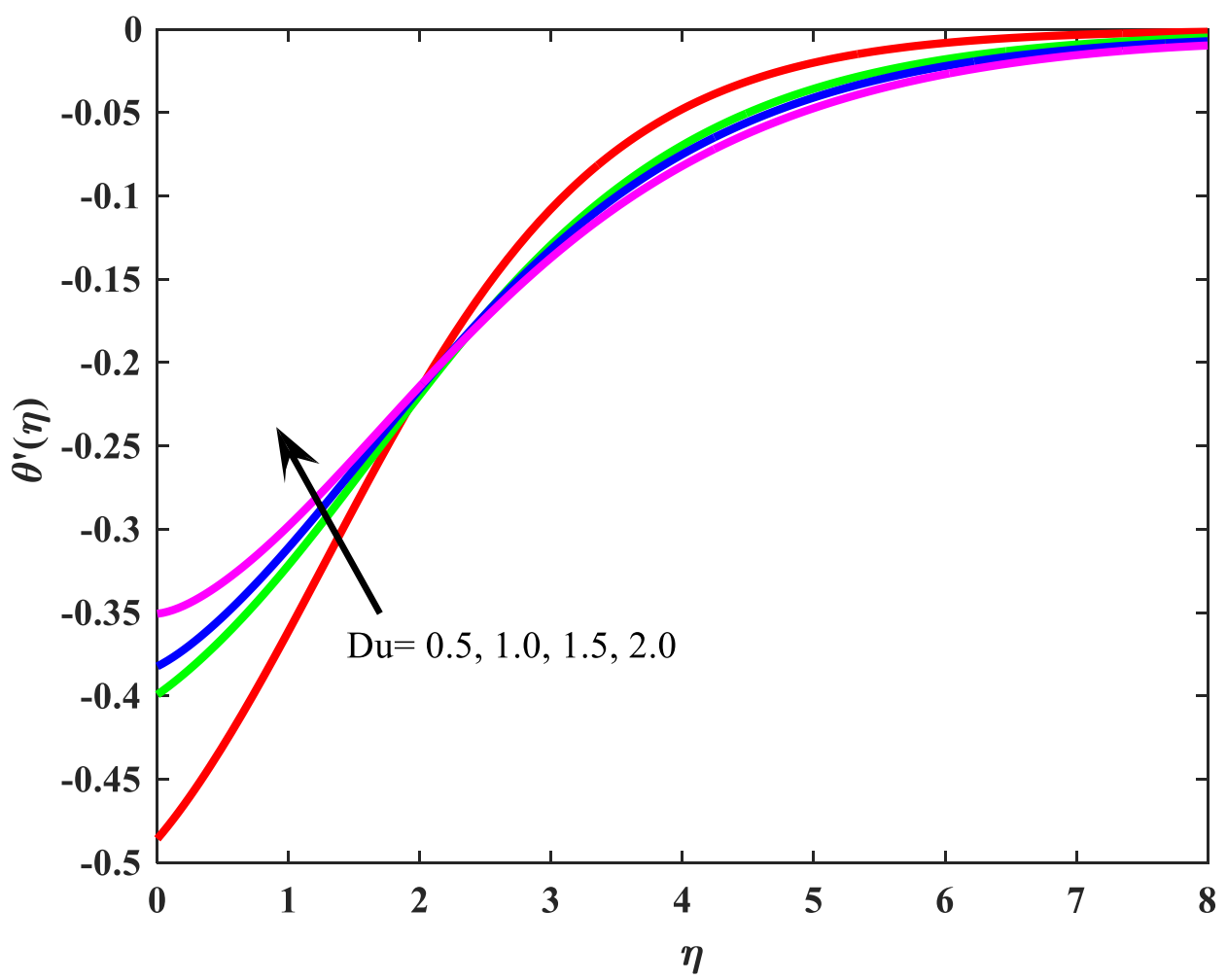

Figure 10(b)-Variations of $D u$ on $\theta^{\prime}(\eta)$ 


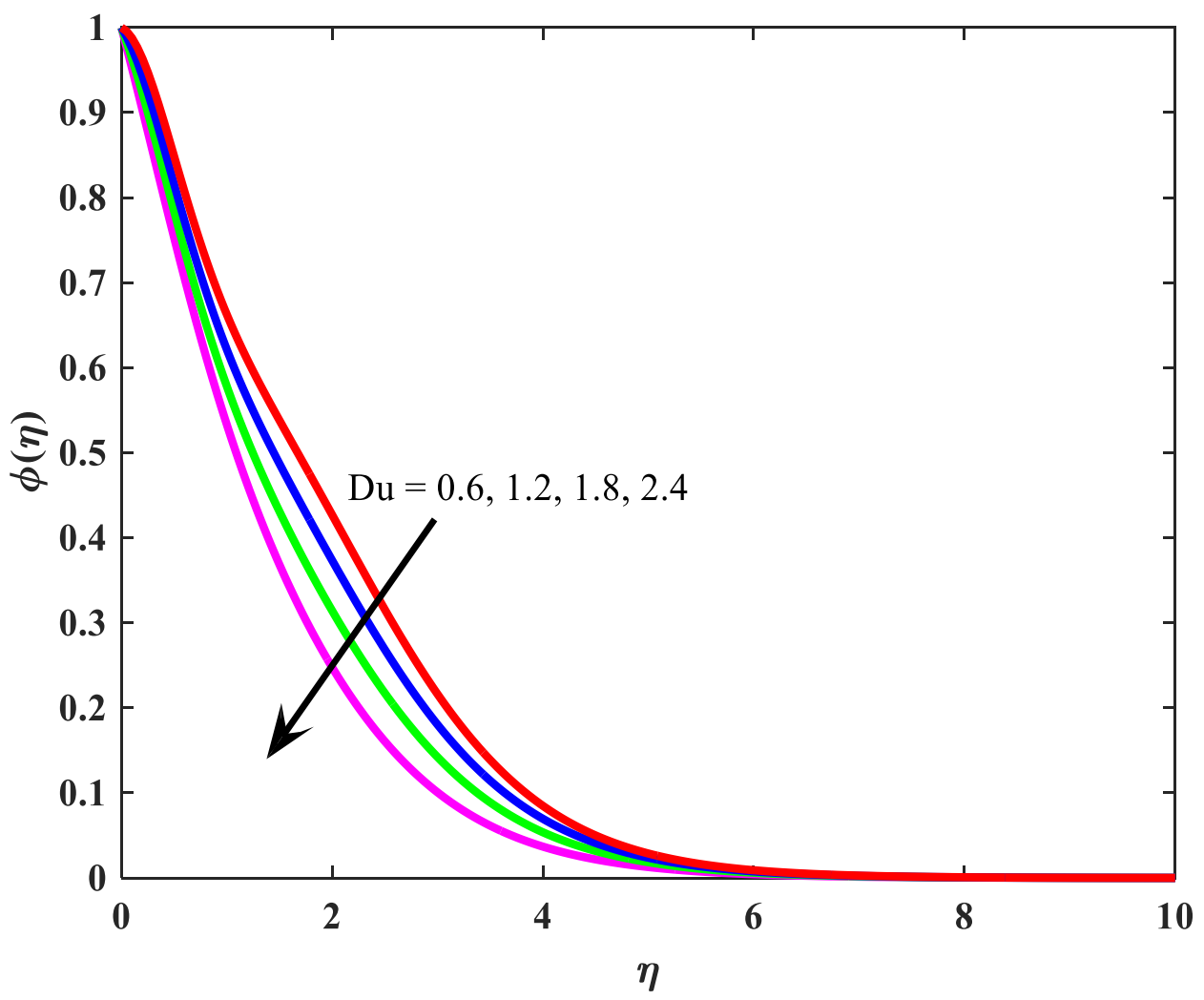

Figure 10(c)-Variations of $D u$ on $\phi(\eta)$

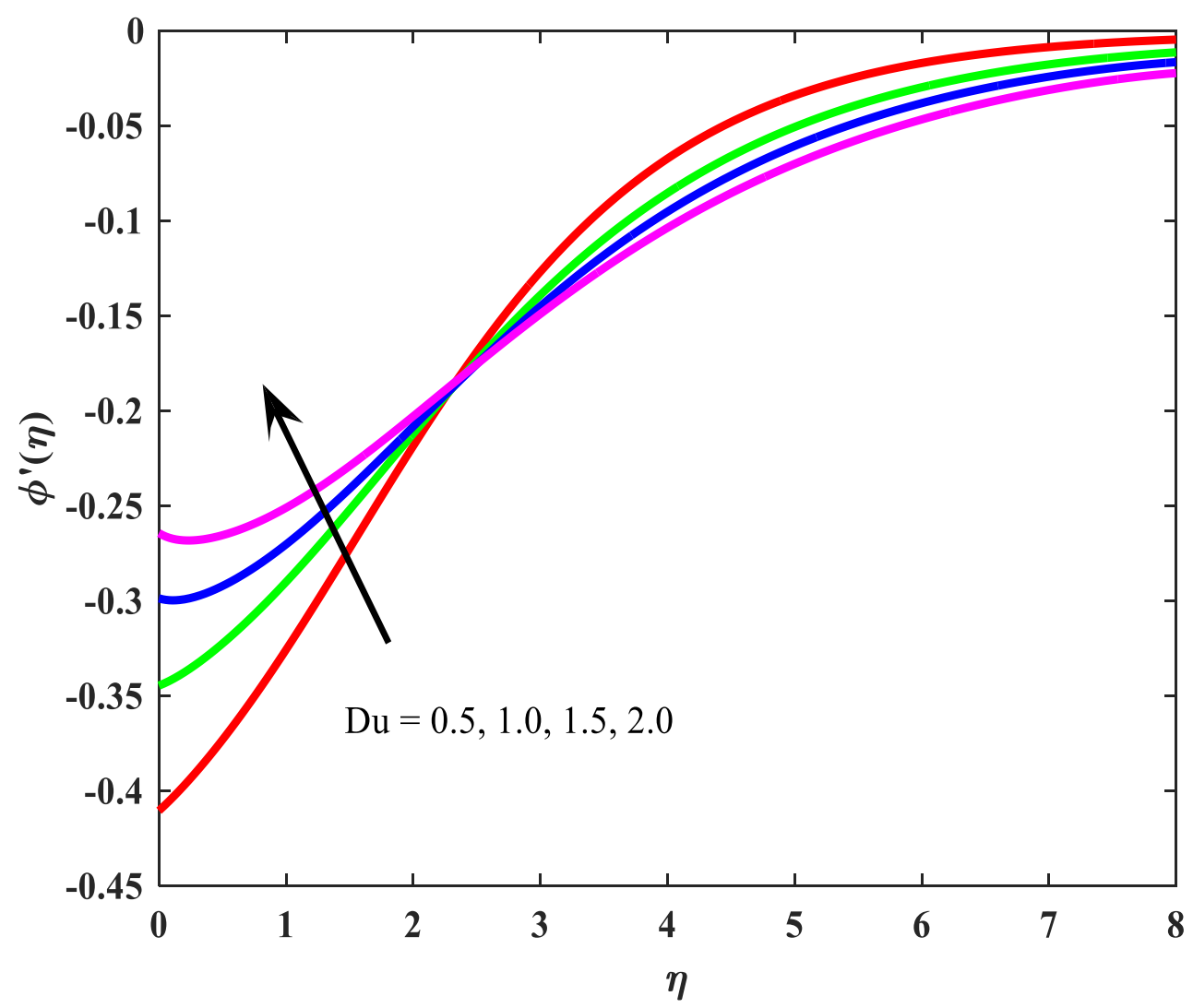

Figure 10(d)-Variations of $D u$ on $\phi^{\prime}(\eta)$ 


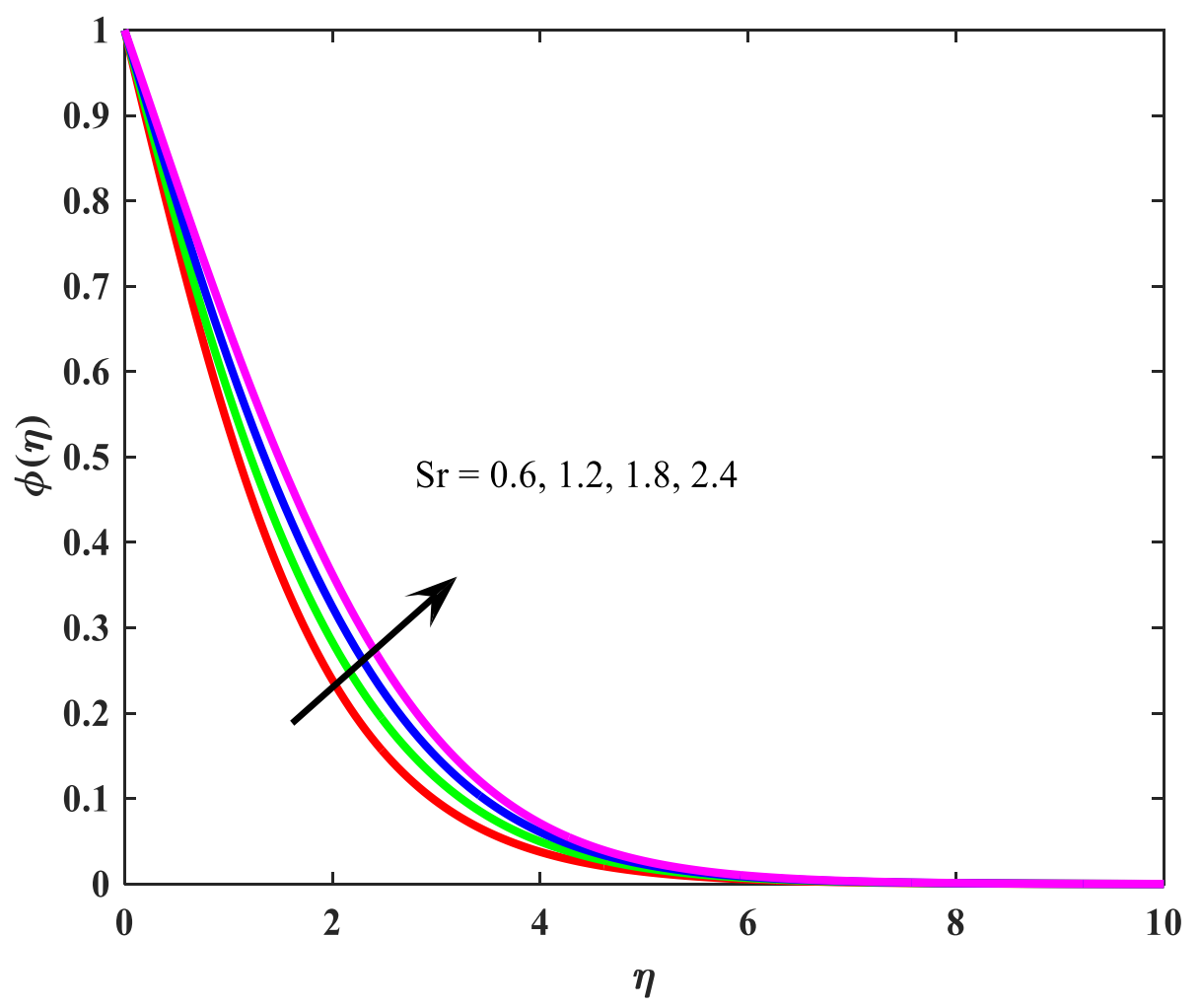

Figure 11(a)-Variations of $S r$ on $\phi(\eta)$

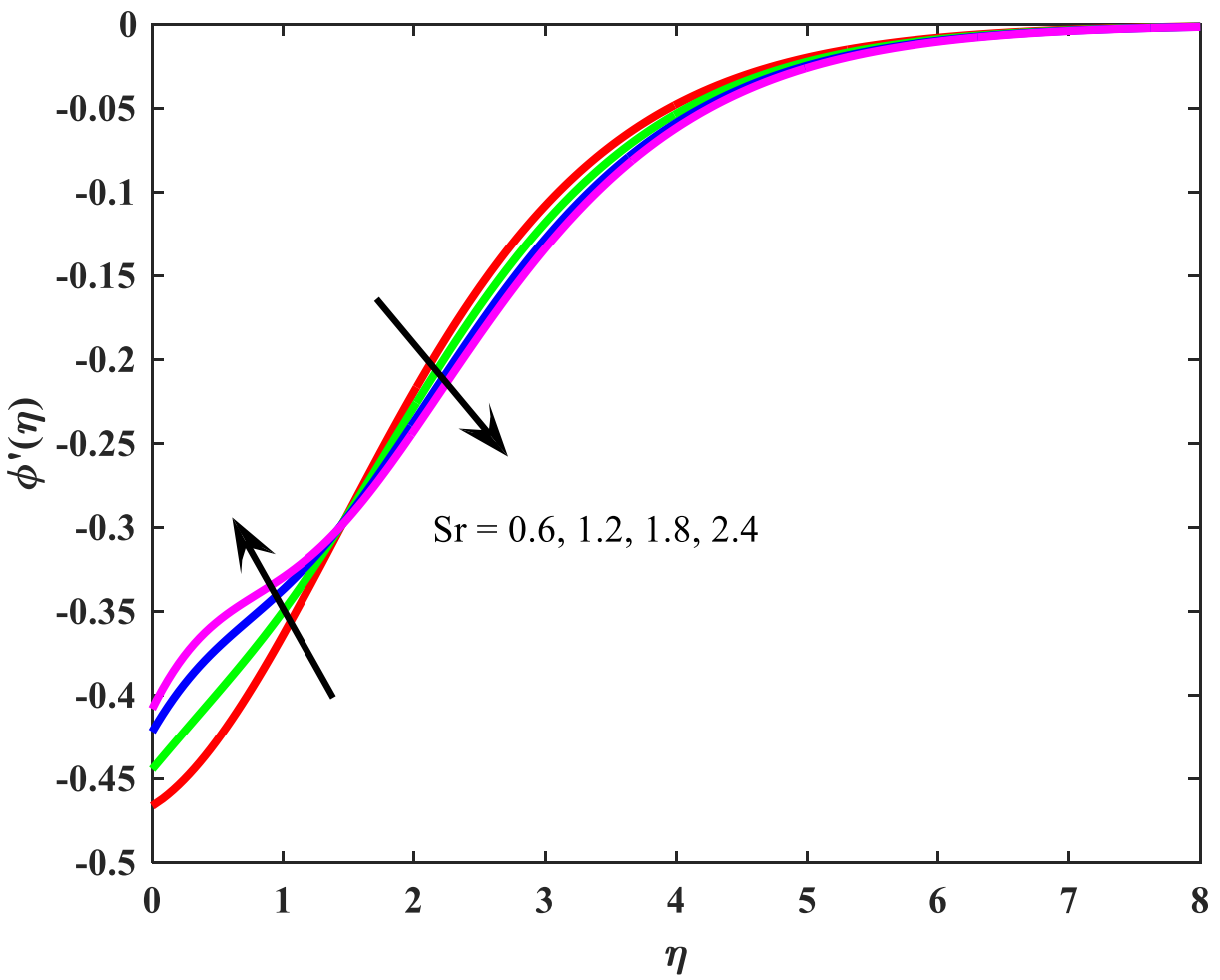

Figure 11(b)-Variations of $S r$ on $\phi^{\prime}(\eta)$

\section{Concluding remarks}

The effects of Cattaneo-Christov heat flux, cross diffusion effects, thermal radiation and chemical reaction on MHD micropolar fluid flow through a paraboloid are examined in the present paper. The 
fourth order R-K method as well as the shooting technique was applied to solve nonlinear ODE's of the problem. The consequences of few flow parameters on the skin friction coefficient to the surface are discussed and the profiles of momentum, heat and mass are graphically illustrated for different flow parameters.

The main findings from our analysis involve a significant increase in the perpendicular velocity at the free stream with a parallel velocity expand near the surface when $G r_{b}$ rises, followed by the reverse trend upon the accumulation of $R_{b}$. Temperature gradient is an increasing function for the raising values of $\mathrm{Nr}$ and $\mathrm{Du}$, while it is a decreasing function with higher values of $\operatorname{Pr}$ and $\gamma$ at an adjacent to the plate, but the effect is the opposite at the point far away from the plate. Concentration profile is increased with increase of Soret effect and reduced when Schmidt number is increased. As a last observation Hartman number and micro rotation parameter have an increasing influence on shear stress.

\section{References}

1. Eringen, A. C. 1966. Theory of micropolar fluid. J. Math. Mech., 16: 1-18.

2. Das, K. 2011. Effect of chemical reaction and thermal radiation on heat and mass transfer flow of MHD micropolar fluid in a rotating frame of reference. International Journal of Heat and Mass Transfer, 54: 3505-3513. doi:10.1016/j.ijheatmasstransfer.2011.03.035

3. Dulal Pal and Sewli Chatterjee. 2010. Mass transfer in MHD non-Darcian flow of a micropolar fluid over a stretching sheet embedded in a porous media with non-uniform heat source and thermal radiation. Communications in Nonlinear Science and Nonlinear Simulation, 15(7): 18431857. https://doi.org/10.1016/j.cnsns.2009.07.024

4. Gangadhar, K., Lakshmi Narayana, K., Sathies Kumar, P., and Rushi Kumar, B. 2017. MHD micropolar fluid flow over a stretching permeable sheet in the presence of thermal radiation and thermal slip flow: a numerical study. Materials Science and Engineering, 263(6): 062010. DOI: $10.1088 / 1757-899 X / 263 / 6 / 062010$

5. Ghadikolaei, S.S., Hosseinzadeh, Kh., Yassari, M., Sadeghi, H., and Ganji, D.D. 2017. Boundary layer analysis of micropolar dusty fluid with $\mathrm{TiO} 2$ nanoparticles in a porous medium under the effect of magnetic field and thermal radiation over a stretching sheet. Journal of Molecular Liquids, 244: 374-389. http://dx.doi.org/10.1016/j.molliq.2017.08.111

6. Gul, T., Afridi, S., Ali, F., Khan, I., and Alshomrani, A.S. 2018. Heat Transmission in the Liquid Film Flow of Micropolar Fluid in a Porous Medium over a Stretching Sheet with Thermal Radiation. Journal of Nano Fluids, 8: 316-324.

7. Mishra, S.R., Khan, I., Al-mdallal, Q.M., and Asifa, T. 2018. Free convective micropolar fluid flow and heat transfer over a shrinking sheet with heat source. Case Studies in Thermal Engineering, 11: 113-119. https://doi.org/10.1016/j.csite.2018.01.005

8. Qasim, M., Khan, I., and Shafie, S. 2013. Heat Transfer in a Micropolar Fluid over a Stretching Sheet with Newtonian Heating. PLoS ONE, 8(4): e59393. https://doi.org/ 10.1371 /journal. pone. 0059393

9. Aurangzaib, Sharif Uddin Md, Bhattacharyya, K., and Shafie, S. 2016. Micropolar fluid flow and heat transfer over an exponentially permeable shrinking sheet. Propulsion and Power Research, 5(4): 310-317. http://dx.doi.org/10.1016/j.jppr.2016.11.005

10. Kumaran, G., Sandeep, N., and Animasaun, I. L. 2017. Computational modeling of magnetohydrodynamic non-Newtonian fluid flow past a paraboloid of revolution. Alexandria Engineering Journal, 57(3): 1859-1865. https://doi.org/10.1016/j.aej.2017.03.019

11. Mair Khan, ArifHussain, Malik, M.Y., and Salahuddin, T. 2017. Biconvection flow of Carreau fluid over an upper paraboloid surface: A computational study. Results in Physics, 7: 4050-4057. https://doi.org/10.1016/j.rinp.2017.10.023

12. Animasaun, I.L., and Pop, I. 2017. Numerical exploration of a non-Newtonian Carreau fluid flow driven by catalytic surface reactions on an upper horizontal surface of a paraboloid of revolution, buoyancy and stretching at the free stream. Alexandria Engineering Journal, 56: 647-658. http://dx.doi.org/10.1016/j.aej.2017.07.005

13. Abegunrin, O.A., Okhuevbie, S.O., and Animasaun, I.L. 2016. Comparison between the flow of two non-Newtonian fluids over an upper horizontal surface of paraboloid of revolution: Boundary 
layer analysis. Alexandria Engineering Journal, 55: 1915-1929. http://dx.doi.org/ 10.1016/j.aej. 2016.08.002

14. Animasaun, I.L. 2016. $47 \mathrm{~nm}$ alumina-water nano fluid flow with in boundary layer formed on upper horizontal surface of paraboloid of revolution in the presence of quadratic autocatalysis chemical reaction. Alexandria Engineering Journal, 55: 2375-2389. http://dx.doi.org/ 10.1016/j. aej.2016.04.030

15. Khalil Ur Rehman, Malik, M.Y., Iffat Zehra, and Alqarni, M.S. 2019. Group theoretical analysis for MHD flow fields: a numerical result. Journal of the Brazilian Society of Mechanical Sciences and Engineering, 41(3): 156. DOI: 10.1007/s40430-019-1662-6.

16. Vishnu Ganesh, N., Quasem M Al-Mdallal, and Kameswaran, P.K. 2019. Numerical study of MHD effective Prandtl number boundary layer flow of $\gamma \mathrm{A} 12 \mathrm{O} 3$ nanofluids past a melting surface. Case Studies in Thermal Engineering, 13: 100413. https://doi.org/10.1016/j.csite.2019.100413

17. Khalil Ur Rehman, Muhammad Awais, Arif Hussain, and Nabeela Kousar. 2019. Mathematical analysis on MHD Prandtl-Eyring nanofluid new mass flux conditions. Mathematical Methods in the Applied Sciences, 42(1): 24-38. DOI: 10.1002/mma.5319

18. Khalil Ur Rehman, Aqeela Qaiser, Malik, M.Y., and Ali, U. 2017. Numerical communication for MHD thermally stratified dual convection flow of Casson fluid yields by stretching cylinder. Chinese Journal of Physics, 55(4): 1605-1614. http://dx.doi.org/10.1016/j.cjph.2017.05.002

19. Ramzan, M., Bilal Jae, M., and Dong Chung, J.2017. Effects of thermal and solutal stratification on Jeffrey magneto-nanofluid along an inclined stretching cylinder with thermal radiation and heat generation/absorption. International journal of mechanical sciences, 131-132: 317-324.http: //dx. doi.org/10.1016/j.ijmecsci.2017.07.012

20. Cattaneo, C. 1948. Sulla conduzionedelcalore, Attidel Seminario Matematicoe Fisico. Dell Universita di Modena e Reggio Emilia, 3: 83-101. DOI: 10.1007/978-3-642-11051-1_5

21. Christov, C.I. 2010. On frame indifferent formulation of the Maxwell-Cattaneo model of finitespeed heat conduction. Mech. Res. Commun., 36: 481-486. DOI: 10.1016/j.mechrescom. 2008. 11.003

22. Straughan, B.2010. Thermal convection with the Cattaneo-Christov model. Int. J. Heat Mass Trans., 53(1-3): 95-98. doi:10.1016/j.ijheatmasstransfer.2009.10.001

23. Hayat, T., Arsalan Aziz, Taseer Muhammad, and Ahmed Alsaedi. 2018. Three-dimensional flow of Prandtl fluid with Cattaneo-Christov double Diffusion. Results in Physics, 9: 290-296. https://doi.org/10.1016/j.rinp.2018.02.065

24. Mamatha Upadhya, S., Raju, C.S.K., Mahesha, and Saleem, S. 2018. Nonlinear unsteady convection on micro and nanofluids with Cattaneo-Christov heat flux. Results in Physics, 9: 779786. https://doi.org/10.1016/j.rinp.2018.03.036

25. Ramzan, M., Bilal, M., and Dong Chung, J. 2017. Soret and Dufour Effects on Three Dimensional Upper-Convected Maxwell Fluid with Chemical Reaction and Non-Linear Radioactive Heat Flux. International Journal of Chemical Reactor Engineering, 15. DOI: 10.1515/ijcre-2016-0136

26. Ramzan, M., Bilal, M., and Dong Chung, J. 2017. Influence of homogeneous-heterogeneous reactions on MHD 3D Maxwell fluid flow with Cattaneo-Christov heat flux and convective boundary condition. Journal of Molecular liquid, 230: 415-422. doi: 10.1016/j.molliq.2017.01.061

27. Ramzan, M., Bilal, M., and Dong Chung, J. 2017. MHD stagnation point Cattaneo-Christov heat flux in Williamson fluid flow with homogeneous-heterogeneous reactions and convective boundary condition-A numerical approach. Journal of Molecular liquid, 225: 856-862. http://dx.doi.org/10.1016/j.molliq.2016.10.139

28. Gnaneswara Reddy, M. 2018. Cattaneo-Christov heat flux effect on hydromagnetic radio active Oldroyd-B liquid flow across a cone/wedge in the presence of cross-diffusion. The European Physical Journal Plus, 133: 24. DOI 10.1140/epjp/i2018-11844-0

29. Mustafa, M.2015. Cattaneo-Christov heat flux model for rotating flow and heat transfer of upper convected Maxwell fluid. AIP Advances, 5: 047109.doi.org/10.1063/1.4917306

30. Gnaneswara Reddy, M. 2015. Influence of thermal radiation and chemical reaction on MHD flow, heat and mass transfer over a stretching surface. Procedia Engineering, 127: 1315-1322. doi: 10.1016/j.proeng.2015.11.489 
31. Hayat, T., Farooq, M., Alsaedi, A., Al-solamy, F., and Al-Solamy, F. 2016. Impact of CattaneoChristov heat flux in the flow over a stretching sheet with variable thickness.AIP Advances, 5: 087159. https://doi.org/10.1063/1.4929523.

32. Waqas, M., Hayat, T., Farooq, M., Shehzad, S.A., and Alsaedi, A. 2016. Cattaneo-Christov heat flux model for flow of variable thermal conductivity generalized Burgers fluid. Journal of Molecular liquid, 220: 642-648. doi.org/10.1016/j.molliq.2016.04.086

33. Rudraswamy, N.G., Ganesh Kumar, K., Gireesha, B.J., and Gorla, R.S.R. 2016. Soret and Dufour effects in three-dimensional flow of Jeffery nanofluid in the presence of nonlinear thermal radiation. Journal of Nanoengineering and Nano manufacturing, 6(4): 1-10.

34. Gnaneswara Reddy, M., and Gorla, R.S.R. 2017. Micropolar fluid flow over a nonlinear stretching convectively heated vertical surface in the presence of Cattaneo-Christov heat flux and viscous dissipation. Frontiers in Heat and Mass Transfer (FHMT), 8(20): 1-9. DOI: 10.5098/hmt.8.20

35. Animasaun, I.L., and Sandeep, N. 2016. Buoyancy induced model for the flow of $36 \mathrm{~nm}$ aluminawater nanofluid along upper horizontal surface of paraboloid of revolution with variable thermal conductivity and viscosity. Powder Technol., 301: 858-867. http://dx.doi.org/10.1016/j.powtec. 2016.07.023

36. Kalyani, K., Seshagiri Rao, N., Makinde, O.D.M., Gnaneswara Reddy, M., and Sudha Rani, M.V.V.N.L. 2019. Influence of viscous dissipation and double stratification on MHD Oldroyd-B fluid over a stretching sheet with uniform heat source. $S N$ Applied Sciences, 1: 334, doi.org/10.1007/s42452-019-0339-1

37. Prasannakumara, B.C., Gnaneswara Reddy, M., Sudha Rani, M.V.V.N.L., and Krishnamurthy, M.R. 2018. Effect of Chemical Reaction on Maxwell Nanofluid Slip Flow over a Stretching Sheet. International Journal of Chemical Reactor Engineering, 17: 217-229. DOI: 10.1515/ijcre-20180065

38. Dheia, G. Salih Al-Khafajy. 2020. Radiation and Mass Transfer Effects on MHD Oscillatory Flow for Carreau Fluid through an Inclined Porous Channel. Iraqi Journal of Science, 61(6): 14261432, DOI: 10.24996/ijs.2020.61.6.21.

39. Narender, G., Govardhan, K., and Sreedhar Sarma1, G. 2020. Numerical Study of Radioactive Magneto hydrodynamics Viscous Nanofluid Due to Convective Stretching Sheet with the Chemical Reaction Effect. Iraqi Journal of Science, 61(7): 1733-1744, DOI: 10.24996 /ijs. 2020.61.7.22

40. Sundos Bader, Suad Naji Kadhim, and Ahmed. M, Abdulhadi. 2020. Influence of Inclined MHD on Unsteady Flow of Generalized Maxwell Fluid with Fractional Derivative between Two Inclined Coaxial Cylinders through a Porous Medium. Iraqi Journal of Science, 61(7): 1705-1714. DOI: $10.24996 / \mathrm{ijs} .2020 .61 .7 .19$ 